DOI: 10.1002/adfm.((please insert DOI)

Submitted to

\title{
Unraveling the Influence of Lanthanide Ions on Intra- and Inter-Molecular Electronic Processes in $\mathrm{Fe}_{10} \mathrm{Ln}_{10}$ nano-Toruses
}

\begin{abstract}
Amer Baniodeh $^{1,2}$, Yu Liang ${ }^{3}$, Christopher E. Anson ${ }^{2}$, Nicola Magnani ${ }^{1}$, Annie K. Powell $^{1,2 *}$, Andreas-Neil Unterreiner ${ }^{3, *}$, Simon Seyfferle ${ }^{4}$, Michael Slota ${ }^{4}$, Martin Dressel $^{4}$, Lapo Bogani ${ }^{4}$, Karin $\mathrm{Goß}^{4, *}$
\end{abstract}

${ }^{1}$ Prof. Annie K. Powell, Dr. Amer Baniodeh, Dr. Nicola Magnani. Institute of Nanotechnology (INT), Karlsruhe Institute of Technology (KIT), P.O Box 76021 Karlsruhe, Germany.

${ }^{2}$ Prof. Annie K. Powell, Dr. Amer Baniodeh, Dr. Christopher E. Anson, Institut für Anorganische Chemie, Karlsruhe Institute of Technology (KIT), Engesserstr. 15, 76131, Karlsruhe, Germany.

${ }^{3}$ PD Dr. Andreas-Neil Unterreiner, Dr. Yu Liang. Institut für Physikalische Chemie, Karlsruhe Institute of Technology (KIT), Fritz-Haber-Weg 2, 76131, Karlsruhe, Germany.

${ }^{4}$ Simon Seyfferle, Michael Slota, Dr. Karin Goß, Dr. Lapo Bogani, Prof. Martin Dressel, 1. Physikalisches Institut, Universität Stuttgart, Pfaffenwaldring 57, 70550, Stuttgart, Germany.

Keywords: lanthanides, electron transport, femtosecond spectroscopy, cyclic coordination clusters, molecular magnetism.

We investigated the electronic properties of the molecular magnetic nanotoruses $\left[\mathrm{Fe}^{\mathrm{III}}{ }_{10} \mathrm{Ln}^{\mathrm{III}}{ }_{10}(\mathrm{Me}-\mathrm{tea})_{10}(\mathrm{Me}-\mathrm{teaH})_{10}\left(\mathrm{NO}_{3}\right)_{10}\right]$, examining the dependence on the lanthanide ( $\mathrm{Ln})$ of both the intra and intermolecular electronic channels. Using femtosecond absorption spectroscopy we show that the intramolecular electronic channels follow a three-step process, which involves vibrational cooling and crossing to shallow states, followed by recombination. A comparison with the energy gaps showed a relationship between trap efficiency and gaps, indicating that lanthanide ions create trap states to form excitons after photo-excitation. Using high-resistance transport measurements and scaling techniques, we investigated the intermolecular transport, demonstrating the dominant role of surface-limited transport channels and the presence of different types of charge traps. The intermolecular transport properties can be rationalized in terms of a hopping model, and a connection is provided to the far-IR spectroscopic properties. Comparison between intra and intermolecular processes highlights the role of the excited electronic states and the recombination processes, showing the influence of Kramers parity on the overall mobility. 


\section{Submitted to

\section{Introduction}

Molecular magnetic materials constitute a thriving field of research, ${ }^{[1]}$ where the interplay of ligands and coordination modes can be used to steer the magnetic properties using chemical means. ${ }^{[2]}$ While most of the first observations were made on transition metal clusters and mainly for $\mathrm{Mn}_{12} \mathrm{Ac}^{[3]}$, recently much attention has focused on rare-earth based magnetic materials ${ }^{[4]}$ where lanthanide ions are used as spin centers, either exclusively or in conjunction with transition metals. This has several advantages. First of all, some lanthanide cations possess very high magnetic anisotropies, so that magnetic systems with slow relaxation of the magnetization can be created. Perhaps more importantly, the lanthanide ions can be changed without significant structural alterations of the compounds, so that the electronic structure and magnetic anisotropy of the ions can be tuned and their effects investigated. This possibility is particularly interesting when investigating the various electronic properties of molecular magnetic materials. ${ }^{[5]}$

In this context, most attention has been focused on hybrid systems where the conducting part is constituted by a carbon nanostructure, a surface or a quantum dot, since most magnetic molecular materials are poor conductors. The investigation of their conducting properties is thus complicated. On the other hand, several theoretical proposals contemplate the use of molecular multilayers or small crystals of molecular magnets to observe novel spintronic effects. Recent efforts have even shown that high electron flows might not be necessary to observe spin transport, and molecular magnetic materials could thus constitute excellent examples of magnetic materials for pure spin transfer. However, the research field suffers from an almost complete lack of understanding of the transport mechanisms in molecular magnetic clusters, preventing progress in the development of the area. Electric Transport experiments have been performed mainly on the archetypal molecular magnet $\mathrm{Mn}_{12} \mathrm{Ac}$, and an understanding of the role of intra- and intermolecular electronic processes is still missing. ${ }^{[6]}$

Here we investigate the role of intra- and intermolecular electronic processes in molecular magnetic clusters, by studying a family of cyclic coordination clusters containing lanthanides and transition metal ions $\left[\mathrm{Fe}_{10} \mathrm{Ln}_{10}(\mathrm{Me} \text {-tea) })_{10}(\mathrm{Me} \text {-teaH) })_{10}\left(\mathrm{NO}_{3}\right)_{10}\right]$ (in the following denoted as $\mathrm{Fe}_{10} \mathrm{Ln}_{10}$ ), together with suitable reference compounds. ${ }^{[7]}$ We use a combination of femtosecond absorption spectroscopy and transport experiments at high voltages, in order to provide a complete picture of the intra- and intermolecular processes, 
respectively. We first quantify the timescales of intramolecular charge trapping and recombination phenomena, showing that electronic excitation of Fe(III) centers decays via multistep processes with the timescale of the electron-hole dynamics comparable to those of nanostructured iron-oxides. We then move to intermolecular processes, where we show dominant intercluster charge hopping, which can be interpreted in the framework of lowamplitude polaron hopping conductivity. Space-charge limited transport becomes dominant at high voltages, with clear charge trap filling effects. The role of the rare-earth in the transport process is finally investigated, and we show that, contrary to intuitive expectations, both the optical and transport properties show a strong dependence on the lanthanide ion in the clusters.

\section{Results and discussion}

\subsection{Structural and Steady-state Optical Properties}

Structural properties: To investigate electronic effects in molecular magnets we chose the family of cyclic coordination clusters $\left[\mathrm{Fe}_{10} \mathrm{Ln}_{10}(\mathrm{Me}-\mathrm{tea})_{10}(\mathrm{Me}-\mathrm{teaH})_{10}\left(\mathrm{NO}_{3}\right)_{10}\right],(\mathrm{Ln}=\mathrm{Gd}$, Tb, Dy, Ho, Er, Tm, Yb, Lu, Y; Me-teaH ${ }_{3}$ is methyl-triethanolamine, or 1-[N,N-bis(2hydroxyethyl)amino]-2-propanol). The isostructural molecules crystallize in the triclinic space group $\mathrm{P} \overline{1}$, with $\mathrm{Z}=1$, and have a toroidal shape, with $2.2 \mathrm{~nm}$ external and $1.2 \mathrm{~nm}$ internal diameters (Figure 1). The coordination clusters are built up from ten FeLn(Me-tea)(MeteaH) $\left.\left(\mathrm{NO}_{3}\right)\right\}$ repeating units, in which the $\mathrm{Fe}^{\mathrm{III}}$ is chelated by the nitrogen and three oxygens of a (Me-tea) $)^{3-}$ ligand, and the $\mathrm{Ln}^{\mathrm{III}}$ is chelated by both a nitrate and a (Me-teaH) ${ }^{2-}$ ligand. Two deprotonated arms from each triethanolamine ligand form pairs of $\mu$-alkoxo bridges between adjacent metal ions in the ring. We have described the molecular structure of the $\mathrm{Fe}_{10} \mathrm{Yb}_{10}$ analogue in more detail elsewhere. ${ }^{[8]}$ The rings are arranged with extremely little overlap and are well separated in the crystal by lattice $\mathrm{MeCN}$ molecules. There are no molecular-based (e.g. hydrogen bonding) interaction pathways between the rings, and the shortest inter-ring distance is $3.9 \AA$, as measured between two methyl groups while the intermolecular $\mathrm{M} \cdot \cdot \mathrm{M}$ distances are all over $8.8 \AA$. It is thus clear that intermolecular electron channels are extremely limited and inefficient, and that one can make a clear distinction between intra and intermolecular electronic processes.

As homometallic reference samples for time-resolved pump-probe experiments, we used the cyclic coordination clusters $\left[\mathrm{Fe}^{\mathrm{III}}{ }_{6}(\text { tea })_{6}\right] \cdot 6 \mathrm{MeOH}$ (abbreviated as $\left.\mathrm{Fe}_{6}\right)$, $\left[\mathrm{Dy}{ }_{6}{ }_{6}{ }^{\mathrm{III}}(\mathrm{Me}-\right.$ tea $\left.)_{6}\left(\mathrm{NO}_{3}\right)_{6}\right] \cdot 6 \mathrm{MeCN}^{[12]}$ and $\left[\mathrm{Tm}_{6}^{\mathrm{III}}(\mathrm{Me}-\mathrm{teaH})_{6}\left(\mathrm{NO}_{3}\right)_{6}\right] \cdot 6 \mathrm{MeCN}$ (abbreviated as $\mathrm{Dy}_{6}$ and $\mathrm{Tm}_{6}$, 
Submitted to

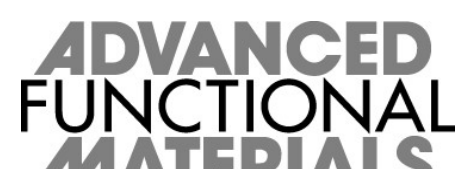

respectively) (Figure 1). Within these clusters, the coordination mode of the (tea) ${ }^{3-}$ or (MeteaH $)^{2-}$ ligands to the $\mathrm{Fe}^{\mathrm{III}}$ or $\mathrm{Ln}^{\mathrm{III}}$ ions, respectively, and their bridges between the metal centers, are similar to those in the $\mathrm{Fe}_{10} \mathrm{Ln}_{10}$ clusters. The synthetic details, characterization and selected crystallographic data of all these compounds are given in Supplementary Information (SI).

Optical properties: UV-Visible spectra (Figure 2) of the compounds including the reference samples show a characteristic broad band centered around $200 \mathrm{~nm}$ with a shoulder extending beyond $300 \mathrm{~nm}$. The spectral features of the $\mathrm{Fe}_{10} \mathrm{Ln}_{10}$ clusters are similar to those of the $\mathrm{Fe}_{6}$ reference compound and also show similarities with reported iron oxide nanoparticles. ${ }^{[9,10,11]}$ According to these earlier studies, the high energy absorption band (200-400 nm) can be assigned mainly to the directly-allowed Ligand to Metal Charge Transfer (LMCT) and partly to the $\mathrm{Fe}^{3+}$ ligand field transitions. ${ }^{[11]}$ Theoretical calculations of one-electron molecular orbitals of $\mathrm{Fe}^{3+}$ oxide and oxyhydroxides showed that the average energy of the quartet ligand field states arise from the $\left(\mathrm{t}_{2 \mathrm{~g}}\right)^{3}\left(\mathrm{e}_{\mathrm{g}}\right)^{2}$ configuration and have been estimated to be located at $325 \mathrm{~nm} \cdot{ }^{[11]}$ Furthermore, transitions originating from lanthanide ions in $\mathrm{Fe}_{10} \mathrm{Ln}_{10}$ clusters can also be observed in the absorption spectra and can be compared with those of the corresponding lanthanide nitrate (Figure S1). Due to the shielding by the outer filled $5 s^{2}$ and $5 p^{6}$ shells, $4 f-4 f$ electronic transitions of trivalent lanthanide ions their absorption spectra features are nearly atom-like with narrow and weak peaks. As a result, we assign the broad absorption band around $300 \mathrm{~nm}$ to LMCT transitions within the iron oxo units in $\mathrm{Fe}_{10} \mathrm{Ln}_{10}$ clusters. The much weaker absorption at $300 \mathrm{~nm}$ in the two reference clusters $\left[\operatorname{Ln}_{6}(\mathrm{Me}-\right.$ teaH $\left.)_{6}\left(\mathrm{NO}_{3}\right)_{6}\right](\mathrm{Ln}=$ Dy and $\mathrm{Tm})$ and $\mathrm{Dy}\left(\mathrm{NO}_{3}\right)_{3}$ as well as the corresponding ligand (Figure S2) indicates different electronic and optical properties. Therefore, these systems were not considered further in this study.

Analysis of the absorption spectra (Figure 2a) reveals a blue-shift of the absorption edge as a function of the various lanthanide ions in the clusters. As a direct consequence, shifts of the optical band gap $\left(E_{\text {opt }}\right)$ of such bulk-like materials with respect to the pure $\alpha$ $\mathrm{Fe}_{2} \mathrm{O}_{3}$ nanoparticle system occur that can be calculated from optical absorption spectra using the Tauc equation: ${ }^{[12]}$

$$
\alpha={ }_{0}\left(-{ }_{\text {opl }}\right)^{n}
$$

where $\alpha$ is the absorption coefficient measured as a function of the photon energy $h v, A_{0}$ is a scale constant, $E_{\text {opt }}$ is the optical band gap energy and, for an allowed direct transition process, 
Submitted to

$n=1 / 2 \cdot{ }^{[13]}$ The resulting plots of $(\alpha h v)^{2}$ vs. $h v$ are shown in Figure 2b, and the resulting intercepts of the tangents to the abscissa (i.e. $h v$ at $\alpha=0$ ) provide direct estimates of the optical band gaps, as summarized in Table 1. The estimated band gap of the $\mathrm{Fe}_{6}$ reference compound was found to be about $(3.48 \pm 0.03) \mathrm{eV}$, which is much larger than that of $\alpha-\mathrm{Fe}_{2} \mathrm{O}_{3}$ nanoparticles $(\sim 2.2 \mathrm{eV}) .{ }^{[14,15]}$ This increase in the band gap can be attributed to quantum confinement effects, which are well known for semiconductors. ${ }^{[16,17]}$ Typical semiconductor band gaps are less than 1-2 eV, while the band gaps of typical insulators are of order 5-10 $\mathrm{eV},{ }^{[18]}$ so the $\mathrm{Fe}_{10} \mathrm{Ln}_{10}$ clusters, with their average band gap energy of $3.75 \mathrm{eV}$, lie between the two. This increase of the optical band gap energy in comparison to the $\mathrm{Fe}_{6}$ cluster is observed in all $\mathrm{Fe}_{10} \mathrm{Ln}_{10}$ clusters.

\subsection{Time-resolved Experiments and Intramolecular Electronic Processes}

To follow the intramolecular photophysical relaxation processes of the iron lanthanide clusters, ultrafast time-resolved transient absorption experiments were performed with an excitation wavelength of $310 \mathrm{~nm}$, i.e. in close resonance to the LMCT state of the iron oxide unit. As can be expected from steady-state absorption spectra (see Figure 2a), this photoexcitation allows for an observation of intramolecular dynamics of the iron oxo units and interactions in the presence of lanthanide ions. In Figure 3a we present femtosecond absorption profiles of these compounds as well as the reference sample in $\mathrm{MeCN} / \mathrm{MeOH}$ solutions. The positive change of the optical density $(\Delta \mathrm{OD})$ at $550 \mathrm{~nm}$ shows multiexponential decay kinetics. Indeed, using a three-exponential fitting routine, three decays were obtained with $\tau_{1}=(200-400) \mathrm{fs}, \tau_{2}$ in the range of a few picoseconds $(1.9-6.7 \mathrm{ps})$, and $\tau_{3}$ on the order of hundreds picoseconds. All time constants and their corresponding relative amplitudes (rel. $A$ ) are defined by

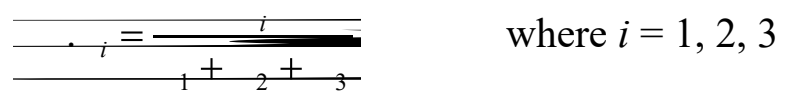

and listed in Table 1. Reference measurements on $\mathrm{Ho}\left(\mathrm{NO}_{3}\right)_{3}, \mathrm{Dy}\left(\mathrm{NO}_{3}\right)_{3}$, and the $\mathrm{Dy}_{6}$ and $\mathrm{Tm}_{6}$ clusters, as well as the pure ligands in solvents, gave no significant pump-induced absorption under the same experimental conditions.

To interpret the three time constants in these compounds, we examined the corresponding temporal profiles of the $\mathrm{Fe}_{6}$ reference cluster and compared them with previously reported ultrafast studies on iron oxide nanoparticles. ${ }^{[16]}$ In the latter work, three time constants $(0.36,4.2$ and $67 \mathrm{ps})$ were found, indicating analogous ultrafast relaxation 
Submitted to processes in $\alpha-\mathrm{Fe}_{2} \mathrm{O}_{3}$ nanoparticles. Studies of ultrafast carrier dynamics in $\alpha-\mathrm{Fe}_{2} \mathrm{O}_{3}$ thin films and single nanocrystals by Joly et al. ${ }^{[19]}$ also suggested that the relaxation of hot electrons to the band edge is completed within $300 \mathrm{fs}$, the recombination or trapping of the holes in $5 \mathrm{ps}$, and the lifetime of trapped states in hundreds of picoseconds. Moreover, femtosecond laser spectroscopic experiments on $\alpha-\mathrm{Fe}_{2} \mathrm{O}_{3}$ nanocrystallite colloidal solutions showed relaxation dynamics for the excited state with three time constants: $\tau_{1}=(142 \pm 30) \mathrm{fs}, \tau_{2}=(4.5 \pm 0.9) \mathrm{ps}$, $\tau_{3}=30 \mathrm{ps}$, while the first relative amplitude rel. $A_{1}=0.65 \cdot{ }^{[20]}$ Similarly, our data on the reference compound ( $\mathrm{Fe}_{6}$ cluster) can be fitted well by a combination of three exponentials, with $\tau_{1}=(190 \pm 15) \mathrm{fs}, \tau_{2}=(5.2 \pm 1.4) \mathrm{ps}, \tau_{3}=(38 \pm 11) \mathrm{ps}$, which nicely agrees with the above mentioned studies. This indicates that the relaxation dynamics are dominated by the iron oxide domain, as already pointed out in section 2.1 (optical properties). Since the iron oxide dynamics may be influenced by the molecular structure, we also studied a non-cyclic ferric oxide compound, $\left[\mathrm{Fe}_{7}^{\mathrm{III}} \mathrm{O}_{3}\left(\mathrm{O}_{2} \mathrm{CCMe}_{3}\right)_{9}(\text { teaH })_{3}\left(\mathrm{H}_{2} \mathrm{O}\right)_{3}\right],{ }^{[21]}$ denoted as $\mathrm{Fe}_{7}$, in which the $(\text { teaH })^{2-}$ ligands have a similar coordination mode to those in the $\mathrm{Fe}_{10} \mathrm{Ln}_{10}$ and $\mathrm{Fe}_{6}$ systems. In $\mathrm{Fe}_{7}$, the dynamics (see Table 1) are, again, close to those of $\alpha-\mathrm{Fe}_{2} \mathrm{O}_{3}$ nanoparticles, but the first decay is found to be almost twice as slow as for the $\mathrm{Fe}_{6}$ cluster (367 and $190 \mathrm{fs}$, respectively). Hence, it can be concluded that the significant difference for the first relaxation time is due to the different molecular structure and that the cyclic $\mathrm{Fe}_{6}$ cluster is indeed a suitable reference system in order to understand the results observed for the cyclic $\mathrm{Fe}_{10} \mathrm{Ln}_{10}$ system.

These femtosecond results indicate, analogously to previous studies on iron oxides, that, after photoexcitation, an exciton-like species undergoes a transition from the $2 p$-valence band orbitals of $\mathrm{O}^{2-}$ to the conduction band (LMCT). ${ }^{[22]}$ This ultrafast process of excited electrons represents the dominant phenomenon ( $\left.\mathrm{rel} . A_{1}=0.61\right)$ and can be equally assigned to vibrational cooling of excited electrons in the conduction band, ${ }^{[16,20,23,23]}$ as outlined below. However, in the presence of the lanthanide ions, the corresponding relaxation times are significantly slower than the pure $\mathrm{Fe}_{6}$ reference sample (Table 1). This indicates that the lanthanide ions create trap states under the band edge of energy gap in the clusters, ${ }^{[24]}$ which can act as a trap to bind either excited electrons or holes. Generally, an impurity increase gives rise to a high density of trap states and, therefore, faster and more efficient trapping of excited electrons into these states. This means that the first decay $\left(\tau_{1}\right)$ is dominated by the competition between vibrational cooling and trapping with high relative amplitude (> 0.61). In agreement with previous reports, the second and third time constants represent the dynamics of recombination and the lifetime of trap states, respectively, with smaller relative 
Submitted to

contributions of rel. $A_{2}+\mathrm{rel} . A_{3}$. The differences in the second and third time constants and their relative amplitudes among the $\mathrm{Fe}_{10} \mathrm{Ln}_{10}$ clusters can also be related to the presence of lanthanide ions. However, we cannot rule out possible small contributions from the effects of lanthanide ions on the carrier dynamics in iron oxide units, either. Although the carrier relaxation time varies slightly among the clusters, the differences are relatively small and irregular. Thus, in the following we will focus our discussion on the ultrafast processes (denoted as $\tau_{1}$ and $\tau_{2}$, resp.).

In order to shed light on the influence of lanthanide ions on the ultrafast dynamics, a simplified picture of the first and second time constants versus the corresponding relative amplitudes is given in Figure 3b. Clearly, lanthanide ions lead to a decrease of the first time constant but also to a higher efficiency (rel. $A_{1}$ ) as compared to the $\mathrm{Fe}_{6}$ cluster. In contrast, the effect of lanthanide ions gives rise to a faster recombination $\left(\tau_{2}\right)$. Despite the shielding effect of the $4 f$ electrons of trivalent lanthanide ions by the outer $5 s^{2}$ and $5 p^{6}$ electrons a weak interaction with their surroundings is still possible, emphasizing the influence of lanthanide ions on ultrafast phenomena like vibrational relaxation, trapping, and recombination.

However, as we will see, it is much more difficult to obtain a quantitative relationship, e.g. between the trivalent lanthanide ion series within the periodic table and their redox potentials. Consider, for example, the dynamics in dependence of Kramers (green in Figure 4) and non-Kramers ions (blue in Figure 4, see also section 2.4). In the case of half-integer spins in trivalent lanthanide ions (Kramers ions) this may be regarded as being of major importance. Significant differences and opposite behaviors were observed for $\mathrm{Fe}_{10} \mathrm{Er}_{10}$ and $\mathrm{Fe}_{10} \mathrm{Tm}_{10}$ clusters, for instance, which also show differences in their magnetism. ${ }^{[2]}$ The time constants and relative amplitudes of $\mathrm{Fe}_{10} \mathrm{Er}_{10}$ are similar to that of the $\mathrm{Fe}_{6}$ cluster (see Table 1), whereas $\mathrm{Fe}_{10} \mathrm{Tm}_{10}$ (and also $\mathrm{Fe}_{10} \mathrm{Eu}_{10}$ ) show the highest rel. $A_{1}=0.88$, indicating efficient trapping. It is worth mentioning that effects on the surroundings of Tm and Er ions were also observed by Ellens et al. ${ }^{[25,26]}$ in a study on the electron-phonon coupling strength of lanthanides in $\mathrm{LiYF}_{4}$-host lattices, using temperature-dependent line broadening measurements, with $\mathrm{Tm}^{3+}$ exhibiting a more efficient interaction with lattice phonons than $\mathrm{Er}^{3+}$. Further explanation of the variation of the electron-phonon coupling strength along the trivalent lanthanide ion series was not given. ${ }^{[26]}$

Next, we briefly want to draw attention to the case of the $\mathrm{Fe}_{10} \mathrm{Y}_{10}$ cluster (see Figure 4), where the ultrafast dynamics behaves exactly opposite to the other $\mathrm{Fe}_{10} \mathrm{Ln}_{10}$ clusters. Indeed, this is a special case, as the absence of $4 d$ - and $4 f$-shell electrons makes $Y(I I I)$, in several ways, closer to a transition-metal than to a lanthanide ion. ${ }^{[27]}$ 
Submitted to

In line with these considerations, the first time constants and the corresponding relative amplitudes are attributed to electron-trapping processes. An effect that can be associated as related to doping of the electron states of the Fe clusters by the lanthanides, can be observed by blue-shifts of the optical absorption edge in the UV/Vis absorption spectrum. As a consequence of these considerations, a relationship between the pump-probe and steadystate processes is proposed. As shown in Figure $\mathbf{4 b}$, comparison of the optical energy gaps $\left(E_{\mathrm{opt}}\right)$ and the first relative amplitudes $\left(\mathrm{rel} . A_{1}\right)$ exhibits indeed a nice correlation, and directly indicates that the larger the optical energy gap, the more efficient the trapping process. Note that the excitonic relaxation dynamics might also cause exciton-exciton annihilation, when two or more excitons are generated in an iron oxide unit. Therefore, we recorded pumpintensity dependent transients at a probe wavelength of $550 \mathrm{~nm}$ in order to investigate possible interactions between the LMCT units. Figure 5 shows the corresponding transients for $\mathrm{Fe}_{10} \mathrm{Er}_{10}$, showing that the decay dynamics are linear with excitation power $(0.4$ to $1 \mu \mathrm{J})$ both at 0.5 and 5 ps delay times. This result suggests that single-exciton annihilation prevails (one exciton per unit), and exciton-exciton annihilation can be excluded. Moreover, the behavior is wavelength-independent (see Figure S3), again in good agreement with the model and previous investigations on iron oxide non-molecular nanostructures. ${ }^{[16]}$

\subsection{Transport Experiments and Intermolecular Electronic Processes}

To investigate intermolecular effects, transport experiments were then performed on the compounds for which suitable single crystals could be obtained. Two-point measurements with a high-impedance setup were performed. The crystals were placed onto n-doped $\mathrm{Si}$ wafers with $300 \mathrm{~nm} \mathrm{SiO}$ layer on top, and contacted using tungsten tips with a tip radius of $10 \mu \mathrm{m}$. The current $I$ could be monitored with a sensitivity of $20 \mathrm{fA}$, as function of the sourcedrain voltage $V$ (with voltages up to $1 \mathrm{kV}$ ) or of the gate voltage $V_{g}$. The transport characteristics were investigated for several crystals for each compound, so as to ensure the reproducibility and check the variability among different crystal qualities and batches. A sample of the observed characteristics is reported in Figure 6a for several $\mathrm{Fe}_{10} \mathrm{Y}_{10}$ crystals. All curves clearly exhibit two regimes with a linear and a non-linear response at low and high voltages, respectively. Both regimes are symmetrically placed around zero voltage and, while at low voltages all curves are very well reproduced from crystal to crystal, at high voltages, the absolute $I$ values may vary appreciably.

The well-reproduced linear response at low voltages can be immediately assigned to standard Ohmic behavior. Below the threshold voltage , where the non-linearity sets in, the 
Submitted to

Ohmic current density is given by Ohm's law neglecting diffusive contributions to the current:

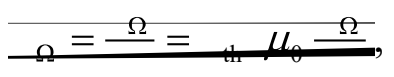

where the subscript $\Omega$ denotes the Ohmic regime, th is the thermal-equilibrium carrier density, $W$ and $L$ are the width and the length of the electronic channel, respectively, and $\mu_{0}$ the charge carrier mobility at low electric fields. From the linear regime in the $I$ - $V$-curves we can then evaluate the sheet resistance $\Omega_{s}=\Omega_{\Omega} \cdot{ }_{\Omega}^{-1} \cdot{ }^{-1}$, where we take $W=20 \mu \mathrm{m}$ as the tip diameter contacting the crystal and $L=200 \mu \mathrm{m}$ is the distance between the tips for each crystal, as determined during the contacting process. For all rare-earths, we obtain extremely high sheet resistances (in the $T \Omega$ range, see Table 2) with a trend, on changing the rare-earth, that is similar to the minimum mobilities (see below). This already provides important information on the transport mechanism present in the $\mathrm{Fe}_{10} \mathrm{Ln}_{10}$ family. The high sheet resistance obtained indicates localized transport processes taking the major role in intermolecular transport rather than delocalized ones. In order to corroborate this observation, the carrier mobility, which can be derived from the measurements as shown below, is the decisive parameter to distinguish between the transport possible mechanisms. On the other hand, the non-linear behavior at high voltages is more complex and more intriguing. Insulating ${ }^{[28]}$ crystals, organic semiconductors (both in crystalline ${ }^{[29]}$ and film ${ }^{[30,31]}$ forms) and even semiconducting nanowires ${ }^{[32,33,34]}$ may display more complex transport phenomena such as space-charge limited (SCL) currents. SCL transport arises when the contacts to the electron reservoirs are Ohmic and the material is not a good conductor, so that at high voltages, an excess of free carriers is injected into the material in the vicinity of the contact. In this situation, the current through the material is no longer limited by its resistance, but by the accumulated space charge, which clogs the conductance channel. As a result, SCL currents allow the investigation of many intrinsic properties of the material that are not easily available in the standard Ohmic regime, such as charge carrier mobility, thermal-equilibrium carrier concentration and charge trap densities. In the present case, due to both the high resistance of the bulk material and the contact geometry, the system will follow surface SCL currents, ${ }^{[35,36]}$ rather than bulk SCL ${ }^{[37,38]}$ (which would only be possible for contacts on opposite sides of a very extended crystal slab). The main characteristic of SCL currents is the presence of a noninjection-limited regime after the Ohmic regime, i.e. above the crossover voltage , where $I$ depends on $\propto^{2}$, with the proportionality constant depending on the contact geometry. In 
Submitted to

our case, where two electric probing tips contact the molecular crystal at the same crystal surface, the relationship for surface SCL currents is ${ }^{[36,37]}$ :

$$
=\frac{2}{\pi} \mu \varepsilon_{0} \varepsilon_{r}-{ }_{2}^{2},
$$

where $\varepsilon_{r}$ is the dielectric constant of the material and $\mu$ is the minimum carrier mobility, which includes the contact quality and the presence of traps in the electron channel. The possible variations in the contact and channel characteristics thus have a huge effect on the absolute current values in this regime, explaining the observed crystal-to-crystal variability in Figure 6a. Small curve asymmetries for positive and negative voltages are also consistent with this picture, as they are usually ascribed to different hole and electron trapping potentials.

A common way to identify the presence of SCL current is by plotting the $I / V$ curve vs $V$, which will yield a straight line in the SCL regime. All compounds of the $\mathrm{Fe}_{10} \operatorname{Ln}_{10}$ family show this behavior, whatever the rare-earth involved, (Figure 6b) clearly highlighting the presence of SCL currents.

To obtain information on the minimum mobility we then extracted the dielectric constant of the systems by using very low energy spectroscopy in the range $18-23 \mathrm{~cm}^{-1}$, where the optical dielectric constant coincides with $\varepsilon_{r}$ in equation 5 . The value can be accurately extracted by using coherent backwave oscillator sources, which emit at a tunable frequency, and then observing the Fabry-Perot interference pattern in a pressed powder sample of a known thickness. The patterns showed no temperature dependence, within the error limits of the measurement, in the whole range between 300 and $10 \mathrm{~K}$. Fitting the data yielded $\varepsilon_{r}=$ $2.8 \pm 0.1$, with the main source of error being variations in the sample thickness (see $\mathbf{S I}$ ).

Following this treatment, we can extract the minimum mobility for all compounds (Table 2), using $W=20 \mu \mathrm{m}$ and $L=200 \mu \mathrm{m}$ as before. The minimum mobility depends strongly on the rare-earth present in the molecule, suggesting these play an important role in the intermolecular electron transport, and we can classify the systems in four main groups of very different mobility. $\mathrm{Fe}_{10} \mathrm{Y}_{10}$ exhibits, by far, the highest mobility, followed by $\mathrm{Fe}_{10} \mathrm{Dy}_{10}$ and $\mathrm{Fe}_{10} \mathrm{Er}_{10}$, which show similar conductivities. $\mathrm{Fe}_{10} \mathrm{Gd}_{10}$ and $\mathrm{Fe}_{10} \mathrm{Lu}_{10}$ have an even lower mobility and may be grouped together with $\mathrm{Fe}_{10} \mathrm{Ho}_{10}$. Two compounds, $\mathrm{Fe}_{10} \mathrm{Tm}_{10}$ and $\mathrm{Fe}_{10} \mathrm{~Tb}_{10}$, showed extremely high resistances $\left(\mathrm{R}>10^{16} \Omega\right)$ and no conductance could be reliably obtained. Furthermore, all extracted mobilities suggest hopping transport to be the transport mechanism for intermolecular electron transport. The boundary between delocalized and localized transport processes, i.e. between band and hopping transport, is commonly 
Submitted to

considered at mobilities in the range of $0.1-1 \mathrm{~cm}^{2} \mathrm{~V}^{-1} \mathrm{~s}^{-1} \cdot{ }^{[39]}$ For all investigated compounds, extremely low mobilities in the order of $10^{-4}$ to $10^{-7} \mathrm{~cm}^{2} \mathrm{~V}^{-1} \mathrm{~s}^{-1}$ are obtained, indicating that inter-molecular hopping transport is the dominant mechanism. This means that the electrons move by rapidly passing from one molecule to another, where they can then behave as indicated by the transient absorption measurements. (Fig. 7)

More information can be extracted from the voltage threshold, , between the Ohmic and SCL regimes, where we have that ${ }_{\Omega}={ }_{\mathrm{SCL}}$ and we can evaluate the thermal-equilibrium carrier density at the crystal surface by:

$$
\text { th }=\frac{2}{\pi} \underline{\underline{\varepsilon_{0} \varepsilon_{r}} \stackrel{c}{\underline{c}} .} .
$$

To extract the threshold voltage we can plot the current in both the Ohmic and the SCL regimes in a double logarithmic plot and then fit the curves with the function $=. \quad \eta_{i}$ (Fig. 8), where $i$ stands for the Ohmic ( $\Omega$ ) or charge-limited (SCL) regions and the exponent $\eta$ varies between the two regimes. is then obtained from the intercept between the fits of the high and low voltage regions. The extracted values are given in Table 2, together with the respective carrier densities. All compounds show an effective thermal carrier density in the order of $10^{13} \mathrm{~m}^{-2}$ at the crystal surface, with no dependence on the rare-earth ion and only small variations observable, in perfect compatibility with the expected variability in contact quality.

As shown in Fig. 9 and the supplementary material, a few measurements show appreciable deviations from the purely quadratic $I$ - $V$-dependence. It has been shown, both experimentally and theoretically ${ }^{[30,40]}$, that surface traps strongly influence the SCL regime. In the case of molecular crystals, traps are often created at the crystal-contact interface at defective molecular sites. In the present case, by using a statistical approach with many measurements, we can identify two main trapping mechanisms taking place (Fig. 9).

The first type of traps, common in organic conducting materials, produces an exponential distribution of electron traps below the Lowest Unoccupied Molecular Orbital (LUMO). In this case, the traps are gradually filled, leading to a current that increases faster than expected for a quadratic dependence, until all trap states are filled. Three-dimensional crystalline semiconductors $^{[41]}$ show $\propto \propto^{m+1} /{ }^{2 m+1}$ for this trapped-charge limited regime, where $d$ is the thickness of the sample, $={ }_{t} /$ and is the characteristic temperature of the traps. Hence exponentially-distributed traps can explain the small deviation from the 
Submitted to

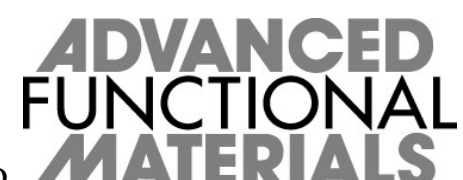

quadratic $I$ - $V$-behavior wherever $B>2$ is observed (e.g. $\mathrm{Fe}_{10} \mathrm{Er}_{10}$ in Fig. 9a). It should be noticed that, possibly due to the low energy of the traps and the surface SCL mechanism, the present systems show far better agreement with the ideal SCL regime than most other molecular conductors: for example in $\mathrm{AlQ}_{3}$, values of $6<m<10$ have been found, with trap energies ${ }_{t} \approx 0.15 \mathrm{eV}^{[42,43]}$. As the present theoretical background considers neither hopping transport nor surface conductance ${ }^{[44]}$, the behavior is best modeled by introducing a fielddependent mobility, without considering a theoretically derived distribution of traps and characteristic temperatures, as already employed for $\mathrm{AlQ}_{3}$ and PPV devices ${ }^{[31,45]}$. We can then assume the mobility to depend on the electric field $E$ via to the Poole-Frenkel mechanism $^{46}$ :

$$
\mu(\quad)=\mu_{0} \exp (-\beta \sqrt{ })
$$

where $\beta=\sqrt{\frac{}{\pi \varepsilon_{0} \varepsilon}}$ and $\mu_{0}$ is the low-field mobility. ${ }^{[1,40,46,47]}$ We can then use the previouslyextracted thermal charge carrier density th to calculate $\mu_{0}$ according to equation (3) and substitute $\mu$ with the Poole-Frenkel mobility $\mu()$ in equation (4). The resulting $I-V$ dependence is drawn in Figure 9a for $\mathrm{Fe}_{10} \mathrm{Er}_{10}$, showing excellent agreement at voltages above $500 \mathrm{~V}$ and an underestimate of the current in the crystal when closer to the crossover.

The second kind of traps that can be observed are localized electron traps, energetically located in the band-gap of the material. ${ }^{[30,39,45]} \mathrm{Fe}_{10} \mathrm{Er}_{10}$ and $\mathrm{Fe}_{10} \mathrm{Dy}_{10}$ show clear signatures of such traps (Figure 9b and c), with clear vertical jumps at the so-called trapfilling voltage ${ }_{\mathrm{TFL}}$. At voltages higher than ${ }_{\mathrm{TFL}}$ transport is in the so-called trap-filled limit and continues to follow the quadratic voltage dependence. In the case of surface SCL currents, these traps are filled by the injected charge at:

$$
\mathrm{TFL}=\frac{\pi}{4 \varepsilon_{0} \varepsilon_{r}} \stackrel{d}{t},
$$

from which the trap density per unit surface area can be evaluated. Here, the index $d$ denotes that we are dealing with deep-traps, located at energies several times from the valence band. This is also visible from the presence of the current jumps due to trap filling, which would otherwise be absent in the presence of a dense distribution of traps close to the conduction band. The presence of such deep traps, which are typically filled close to $0.4 \mathrm{eV}$, is very likely in systems with very wide band-gaps, above $3.5 \mathrm{eV}$, as evaluated from the 
Submitted to

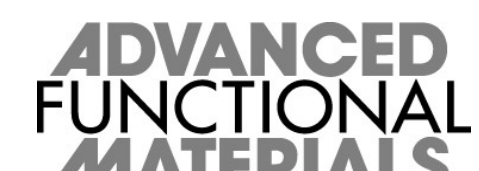

optical spectra (see Figure 2). The typical trap densities observed for the $\mathrm{Fe}_{10} \mathrm{Ln}_{10}$ family lie around $=3.5 \pm 0.3 \cdot 10^{10} \mathrm{~cm}^{-2}$, which compares very favorably to known trap densities in organic conductors, ${ }^{[48]}$ and in particular to experiments on surface-limited charge transport in rubrene single crystals. ${ }^{[49]}$

\subsection{Relations between Intra and Inter-molecular Electronic Processes}

To investigate the relation among inter and intramolecular processes we started by considering that the systems behave as very large band-gap semiconductors, with gaps always above $3.5 \mathrm{eV}$. This means that at room temperature all electrons fill the valence band, with extremely little thermal population of the conduction band, as also confirmed by the transport experiments. As a consequence, the conduction charges injected via the leads will behave in a way similar to the photo-excited electrons, with the additional process of hopping from one molecule to another. As both shallow trap states (where the electrons rest after the $\tau_{1}$ process) and conduction band electrons may contribute to the electron hopping, the relevant parameter is the total electron-hole recombination rate, $\Gamma_{\mathrm{re}}=1 / \tau_{2}+1 / \tau_{3}$. Using this link, we can then compare the transient absorption and transport experiments by plotting $\Gamma_{\mathrm{re}}$ vs the intermolecular charge mobility (Figure 10). The resulting graph evidences much higher mobilities for lower rates, with the rates usually decreasing roughly linearly on increasing the atomic number of the ions. The trend is also distinctly dissimilar for Kramers and for nonKramers ions, with Kramers systems showing much higher mobilities than non-Krames centers at the same electron-hole recombination rate. The increase in the number of electrons per rare-earth produces, for both Kramers and non-Kramers ions, an almost identical decrease in the electron-hole recombination rate, but the resulting mobilities are $c a 1$ order of magnitude higher in Kramers systems.

This analysis presumes that the trapping time $\tau_{1}$ has no influence on the overall transport properties, as both excited and shallow-trapped states can contribute to the hopping and the overall conduction in the system. To check that this is indeed true, we can plot $\tau_{1}$ against the thermal-equilibrium carrier density at the crystal surface for all compounds (Figure 10b). The resulting plot shows, again, two relatively distinct regions (highlighted in blue and green in the figure) where Kramers and non-Kramers ions, respectively, lie, with the Kramers systems having shorter trapping times. On the other hand, there is no visible relation between $n_{\text {th }}$ and the trapping time, confirming the validity of the assumptions. 


\section{Conclusions}

Submitted to

We have investigated electron transport and excitation processes in a series of molecular magnetic toruses, both at the intra- and inter-molecular level. Using time-resolved absorption spectroscopy we showed the details of the intramolecular electron processes and their characteristic times, providing a full picture of the resulting three-level scheme. The relevant processes were identified as vibrational cooling and fast conversion towards shallow states, followed by electron-hole recombination, consistent with the dynamic recombination in iron oxide nanoparticles, but modulated by the presence of the rare-earths.

Using very high-resistance transport measurements we were then able to identify, for the first time, space-charge limited currents in magnetic molecular crystals. Connection to the spectroscopic properties at very low energies provides an accurate estimation of the mobilities and carrier densities. The hopping mechanism from one molecule to another is strongly affected by the presence of traps, and several types of traps were identified with the resulting trap densities comparing favorably to those of well-known molecular conductors.

For both intra- and inter-molecular processes a clear distinction between Kramers and non-Kramers ions is observed with the former showing much higher charge densities and electron mobilities. A correlation between the intra- and intermolecular processes is highlighted, showing no significant role of the trapping dynamics, and the importance of both excited and trapped states to the hopping and intermolecular charge transport.

These observations offer a first insight into the transport processes of molecular magnetic materials. As such, our results can be considered as a first step towards the rationalization and modeling of molecular spintronic materials. In particular, the observed phenomena involving traps, hopping and surface-limited-transport are largely ignored in present theoretical treatments, and should likely be included. The presence of trapping states with lifetimes of hundreds of picoseconds (i.e. longer than spin-flip attempt rates in many materials) and low hopping occurences also hints at the possibility of controlling such magnetic systems using optical pulses, and should stimulate the adapting recently-proposed control schemes ${ }^{[52-54]}$ to magnetic rings. In addition, the combination of optical and transport properties is shown to be extremely fruitful as a means for following the plethora of phenomena affecting charge transport in molecular magnetic materials, and will likely establish the methodological basis for future investigations in the subject.

\section{Experimental Section}


Submitted to

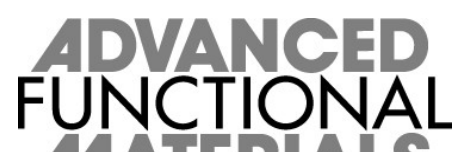

MATERIALS

Synthesis: The syntheses of the $\mathrm{Fe}_{10} \mathrm{Ln}_{10}$ clusters were performed analogously to the synthesis of the previously reported $\mathrm{Fe}_{10} \mathrm{Yb}_{10}$ compound ${ }^{[8]}$ and are given in the $\mathrm{SI}$ in more detail.

Crystallography: Details of the crystal structure determinations are given in the Supplementary Material. Crystallographic data (excluding structure factors) for the structure(s) reported in this paper have been deposited with the Cambridge Crystallographic Data Centre as supplementary publication no. CCDC 885249, 885250, 912850, 984458984462. Copies of the data can be obtained, free of charge, on application to CCDC, 12 Union Road, Cambridge CB2 1EZ, UK:

http://www.ccdc.cam.ac.uk/cgi-bin/catreq.cgi, e-mail: data_request@ccdc.cam.ac.uk, or fax: +441223336033.

Optical measurements: Absorption spectra of the clusters in buffer solution were measured using Cary 500 UV-Vis-NIR spectrophotometer. The samples were measured with reference to a blank containing buffer solution and corresponding ligands in $1 \mathrm{~mm}$ quartz cuvettes (Hellma).

Transient absorption measurements: The time-resolved pump-probe measurements and the experimental setup were as described previously. ${ }^{[50]}$ Femtosecond laser pulses (CPA 2210, Clark-MXR) were generated using a Ti:Sapphire oscillator and amplified with a regenerative amplifier. At the output, these pulses with a repetition rate of $1 \mathrm{kHz}$ were split equally to pump two non-collinear optical parametric amplifiers (NOPAs, Clark-MXR). One of these two output pulses served for sum-frequency generation $(310 \mathrm{~nm}$ with pulse duration about $100 \mathrm{fs})$ via a sum frequency mixing process with the center wavelength of the CPA $(775 \mathrm{~nm})$. The energy of the pump pulses was varied from 0.4 to $1.0 \mu \mathrm{J}$. The probe pulses were varied between 500 and $600 \mathrm{~nm}$ with energy of less than $0.1 \mu \mathrm{J}$. The polarization of the probe beam was rotated to magic angle $\left(54.7^{\circ}\right)$ relative to that of the pump beam in order to avoid anisotropic effects. After passing through a computer controlled optical delay line (Physik Instrumente), the probe pulses were overlapped with the pump pulses and focused into a 1$\mathrm{mm}$ standing quartz cuvette (Hellma) containing the sample cluster solutions. All measurements were carried out at room temperature.

Transport measurements: All transport measurements were conducted on single crystals connected via $\mathrm{W}$ microtips using micromanipulators. The distance between the tips was checked using an optical microscope with in-built crossbar ruler and a resolution of $20 \mu \mathrm{m}$. The high-resistance measurements were performed with a Keithley 6517A system to read the current and apply the source-drain voltage (up to $1 \mathrm{kV}$ ), with $20 \mathrm{fA}$ resolution. All 
Submitted to

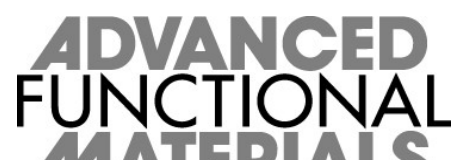

measurement lines were filtered with $65 \mathrm{~dB}$ insertion loss at $1000 \mathrm{MHz}$ using Spectrum Control $\pi$-filters.

THz measurements: The $\mathrm{THz}$ data were acquired using BWO backwave oscillators as monochromatic, coherent sources and a Golay cell as detector, with a synchronous detection by a chopper working at a frequency of $1 \mathrm{kHz}$ internal reflections from the sample surface were used to measure the dielectric constant, as explained in the supplementary material.

\section{Acknowledgements}

This work was funded by the state Baden-Württemberg, the Karlsruhe Institute of Technology (KIT) and the Deutsche Forschungsgemeinschaft (DFG as administrated through the Center for Functional Nanostructures (CFN). We thank the BW-Stiftung (Kompetenznetz Funktionelle Nanostrukturen), the DFG (SPP-TRR21), the Humboldt Stiftung (Sofja Kovalevskaja Prize) and ERC (ERC-StG-338258 "OptoQMol") for financial support.

We acknowledge the ANKA Synchrotron Light Source, Karlsruhe Institute of Technology, for the provision of beamtime for the structure determinations of $\mathrm{Fe}_{10} \mathrm{Dy}_{10}$ and $\mathrm{Fe}_{10} \mathrm{Y}_{10}$, and thank Dr. Gernot Buth for experimental assistance on the SCD beamline.

We are grateful for support from Dr. Konstantis Konidaris, Vanessa Grande and Lena Friedrich.

Received: ((will be filled in by the editorial staff))

Revised: ((will be filled in by the editorial staff))

Published online: ((will be filled in by the editorial staff))

\section{References:}

[1] D. Gatteschi, R. Sessoli, J. Villain, Molecular Nanomagnets (Oxford University Press, Oxford, 2006)

[2] a) A. Mandel, W. Schmitt, T. G. Womack, R. Bhalla, R. K. Henderson, S. L. Heath and A. K. Powell, Coord. Chem. Rev. 1999, 192, 1067; b) R. W. Saalfrank, H. Maid and A. Scheurer, Angew. Chem., Int. Ed. 2008, 47, 8794; c) G. E. Kostakis, I. J. Hewitt, A. M. Ako, V. Mereacre and A. K. Powell, Philos. Trans. R. Soc. A 2010, 368, 1509. 
Submitted to

[3] R. Bagai, G. Christou, Chem. Soc. Rev. 2009, 38, 1011-1026.

[4] N. Ishikawa, M. Sugita, T. Khoshiawa, S. Kaizu, J. Phys. Chem. B 2004, 108, 11265-11271.

[5] a) A. R. Rocha, V. M. García-Suárez, S. W. Bailey, C. J. Lambert, J. Ferrer, S. Sanvito, Nature Mater. 2005, 4, 335; b) L. Bogani, W. Wernsdorfer, Nat. Mater. 2008, 7, 179-186; c) J. Park, A. N. Pascupathy, J. I. Goldsmith, C. Chang, Y. Yaish, J. R. Petta, M. Rinkoski, J. P. Sentha, H. D. Abruna, P. L. McEuen, D. C. Ralph, Nature, 2002, 417, 722; d) H. B. Beersche, Z. de Groot, J. A. Folk, H. S. J. van der Zant, C. Romeike, M. R. Wegewijs, L. Zobbi, D. Barrera, E. Tondello, A. Cornia, Phys. Rev. Lett. 2006, 96, 206801; e) J. E. Grose, E. Tam, C. Timm, M. Scheloske, B. Ulgut, J. J. Parks, H. D. Abruña, W. Harneit, D. C. Ralph, Nature Mater. 2008, 7, 884; f) D. Ruger, R. Budaikian, H. J. Mamin, B. W. Chui, Nature 2004, 430, 329; g) R. Vincent, S. Klyatskaya, M. Ruben, W. Wernsdorfer, F. Balestro, Nature 2012, 488, 357-360; h) C. Romeike, M. R. Wegewijs, W. Hofstetter, H. Schoeller, Phys. Rev. Lett. 2006, 96, 196601; i) M. Misiorny, I. Weymann, J. Barnaś, Phys. Rev. B 2009, 79, 224420; j) S. Lindebaum, D. Urban, J. König, Phys. Rev. B 2010, 81, 024421; k) C. Cervetti, E. Heintze and L. Bogani Dalton Trans. 2014, 43, 4220 .

[6] D. Zippe, J. M. North, R. M. Achey, N. S. Dalal, S. Hill, J. Appl. Phys. 2004, 95, 6900.

[7] A. Baniodeh, in Cooperative effects in non-cyclic and cyclic FeIII/4f coordination clusters, Cuvillier Verlag Göttingen, ISBN 978-3-95404-448-1 (2013).

[8] A. Baniodeh, C. E. Anson, A. K. Powell, Chem. Sci. 2013, 4, 4354-4361.

[9] A. Kebede, A. K. Singh, P. K. Rai, N. K. Giri, A. K. Rai, G. Watal, A. V. Gholap, Lasers Med. Sci. 2013, 28, 579.

[10] Y. P. He, Y. M. Miao, C. R. Li, S. Q. Wang, L. Cao, S. S. Xie, G. Z. Yang, B. S. Zou, C. Burda, Phys. Rev. B 2005, 71, 125411.

[11] D. M. Sherman, T. D. Waite, Am. Mineral. 1985, 70, 1262.

[12] a) J. Tauc, A. Abraham, L. Pajasova, R. Grigorovici, A. Vancu in Non-Crystalline Solids, North-Holland, Amsterdam, 1965, p. 606. b) J. C. Phillips, Excitons in The Optical Properties of Solids (Ed. J. Tauc), Academic, New York, 1966, p. 155. c) D. L. Reid, K. R. Kreitz, M. A. 
Submitted to

Stephens, J. E. S. King, P. Nachimuthu, E. L. Petersen, S. Seal, J. Phys. Chem. C 2011, 115, 10412.

[13] S. T. Navale, D. K. Bandgar, S. R. Nalge, R. N. Mulik, S. A. Pawar, M. A. Chougule, V. B. Patil, J. Mater Sci: Mater Electron, 2013, 24, 1422.

[14] Q. Zhang, S. Dunn, J. Nanosci. Nanotechnol. 2011, 11, 3716.

[15] N. J. Cherepy, D. B. Liston, J. A. Lovejoy, H. Deng, J. Z. Zhang, J. Phys. Chem. B 1998, 102, 770.

[16] S. Baskoutas, A. F. Terzis, J. Appl. Phys. 2006, 99, 013708.

[17] K. Takei, H. Fang, S. B. Kumar, R. Kapadia, Q. Gao, M. Madsen, H. S. Kim, C.-H. Liu, Y.-L. Chueh, E. Plis, S. Krishna, H. A. Bechtel, J. Guo, A. Javey, Nano Lett. 2011, 11, 5008.

[18] J. C. Phillips in Bonds and Bands in Semiconductors, Academic Press, New York and London, 1973, pp. 3.

[19] A. G. Joly, J. R. Williams, S. A. Chambers, G. Xiong, W. P. Hess, J. Appl. Phys. 2006, 99, 053521.

[20] J. Kiwi, N. Denisov, Y. Gak, N. Ovanesyan, P. A. Buffat, E. Suvorova, F. Gostev, A. Titov, O. Sarkisov, P. Albers, V. Nadtochenko, Langmuir, 2002, 18, 9054.

[21] L. F. Jones, P. Jensen, B. Moubaraki, K. J. Berry, J. F. Boas, J. R. Pilbrow, K. S. Murray, J. Mater. Chem. 2006, 16, 2690.

[22] H. M. Fan, G. J. You, Y. Li, Z. Zhang, H. R. Tan, Z. X. Shen, S. H. Tang, Y. P. Feng, J. Phys. Chem. C 2009, 113, 9928.

[23] K. Korobchevskaya, C. George, A. Diaspro, L. Manna, R. Cingolani, A. Comin, Appl. Phys. Lett. 2011, 99, 011907.

[24] P. Dorenbos, J. Phys.: Condens. Matter 2003, 15, 8417.

[25] A. Ellens, H. Andres, M. L. H. Ter Heerdt, R. T. Wegh, A. Meijerink, G. Blasse, Phys. Rev. B $1997,55,180$.

[26] A. Ellens, H. Andres, A. Meijerink, G. Blasse, Phys. Rev. B 1997, 55, 173. 
Submitted to

[27] K. A. Gschneider, L. Eyring in Handbook on the Physics and Chemistry of Rare Earths Vol. 1: Metals, North-Holland Publishing Company, 1978.

[28] R. W. Smith, A. Rose, Phys. Rev. 1955, 97, 1531.

[29] R. W. I. de Boer, M. Jochemsen, T. M. Klapwijk, A. F. Morpurgo, J. Niemax, A. K. Tripathi, J. Pflaum, J. Appl. Phys. 2004, 95, 1196.

[30] A. Ioannidis, E. Forsythe, Yongli Gao, M. W. Wu, E. M. Conwell, Appl. Phys. Lett. 1998, 72, 3038.

[31] M. Stößel, J. Staudigel, F. Steuber, J. Blässing, J. Simmerer, A. Winnacker, Appl. Phys. Lett. 2000, $76,115$.

[32] A. D. Schricker, F. M. Davidson III, R. J. Wiacek, B. A. Korgel, Nanotechnology 2006, 17, 2681.

[33] A. A. Talin, F. Léonard, B. S. Swartzentruber, X. Wang, S. D. Hersee, Phys. Rev. Lett. 2008, $101,076802$.

[34] A. M. Katzenmeyer, F. Léonard, A. A. Talin, M. E. Toimil-Molares, J. G. Cederberg, J. Y. Huang, J. L. Lensch-Falk, IEEE T. Nanotechnol. 2011, 10, 92.

[35] J. A. Geurst, Phys. Status Solidi 1966, 15, 107.

[36] A. A. Grinberg, S. Luryi, M. R. Pinto, N. L. Schryer, IEEE Trans. Electron Dev. 1989, 36, 1162.

[37] A. Rose, Phys. Rev. 1955, 97, 1538.

[38] M. A. Lampert, Phys. Rev. 1956, 103, 1648.

[39] G. Horowitz, Adv. Mat. 1998, 10, 365.

[40] R. W. I. de Boer, A. F. Morpurgo, Phys. Rev. B 2005, 72, 073207.

[41] P. Mark, W. Helfrich, J. Appl. Phys. 1962, 33, 205.

[42] P. E. Burrows, Z. Shen, V. Bulovic, D. M. McCarty, S. R. Forrest, J. A. Cronin, M. E. Thompson, J. Appl. Phys. 1996, 79, 7991.

[43] A. J. Campbell, D. D. C. Bradley, D. G. Lidzey, J. Appl. Phys. 1997, 82, 6326. 
Submitted to

[44] M.A. Lampert, P. Mark, Current Injection in Solids (ISBN 0124353509, Academic Press, New York, 1970).

[45] W. Brütting, S. Berleb, A. G. Mückl, Synt. Met. 2001, 122, 99.

[46] S. M. Sze, K. K. Ng Physics of Semiconductor Devices (Third Edition, John Wiley \& Sons, Hoboken, 2007)

[47] N. Karl, Synt. Met. 2003, 133, 649.

[48] a) V. Coropceanu, J. Cornil, D.A. da Silva Filho, Y. Olivier, R. Silbey, J.-L. Brédas, Chem. Rev. 2007, 107, 926-952; b) J.E. Anthony, Chem. Rev. 2006, 106, 5028-5048; c) Y. Shirota, H. Kageyama, Chem. Rev. 2007, 107, 953-1010; d) J.E. Anthony, Angew. Chem. Int. Ed. 2008, 47, 452-483; e) M.E. Gershenson, V. Podzorov, A.F. Morpurgo, Rev. Mod. Phys. 2006, 78, 973989; f) S.A. DiBenedetto, A. Facchetti, M.A. Ratner, T.J. Marks, Adv. Mat. 2009, 21, 14071433.

[49] V. Podzorov, E. Menard, A. Borissov, V. Kiryukhin, J. A. Rogers, M. E. Gershenson, Phys. Rev. Lett. 2004, 93, 086602.

[50] T. J. A. Wolf, D. Voll, C. Barner-Kowollik, A.-N. Unterreiner, Macromolecules, 2010, 45, 2257.

[52] E. Heintze, F. El Hallak, C. Clauß, A. Rettori, M. G. Pini, F. Totti, M. Dressel, L. Bogani, Nature Materials, 2013, 12, 202.

[53] T. Liu, H. Zheng, S. Kang, Y. Shiota, S. Hayami, M. Mito, O. Sato, K. Yoshizawa, S. Kanegawa and C. Duan, Nat. Commun., 2013, 4, 2826.

[54] M. Y. Tsang, C. Clauß, M. Scheffler, M. Dressel, L. Bogani, Nature Communications, to be published. 


\section{submitted to MATERIAS

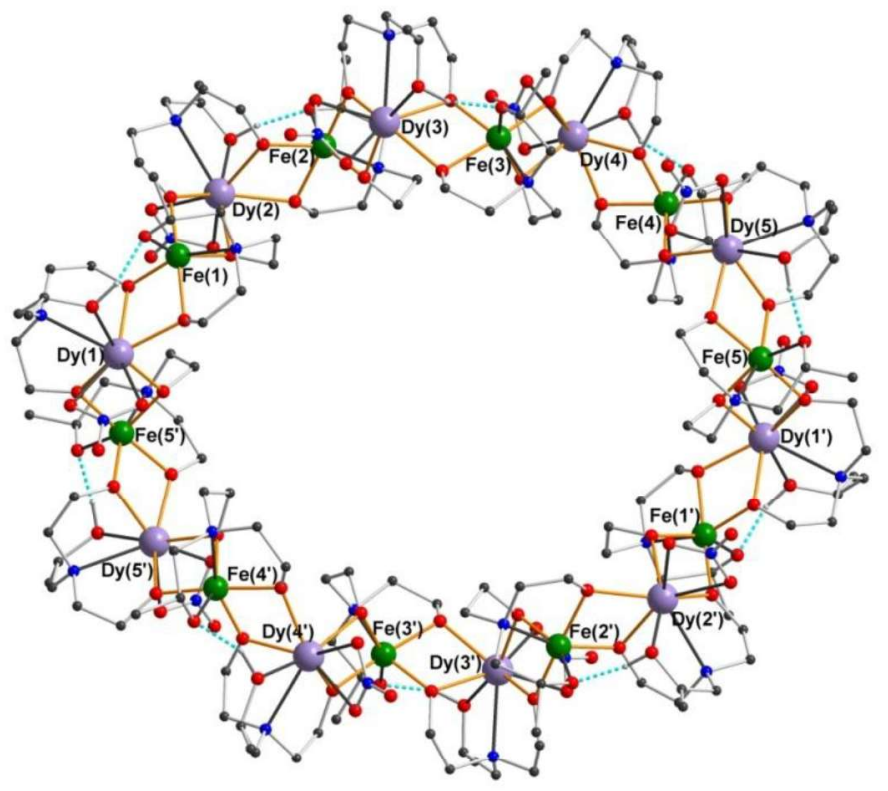

c)
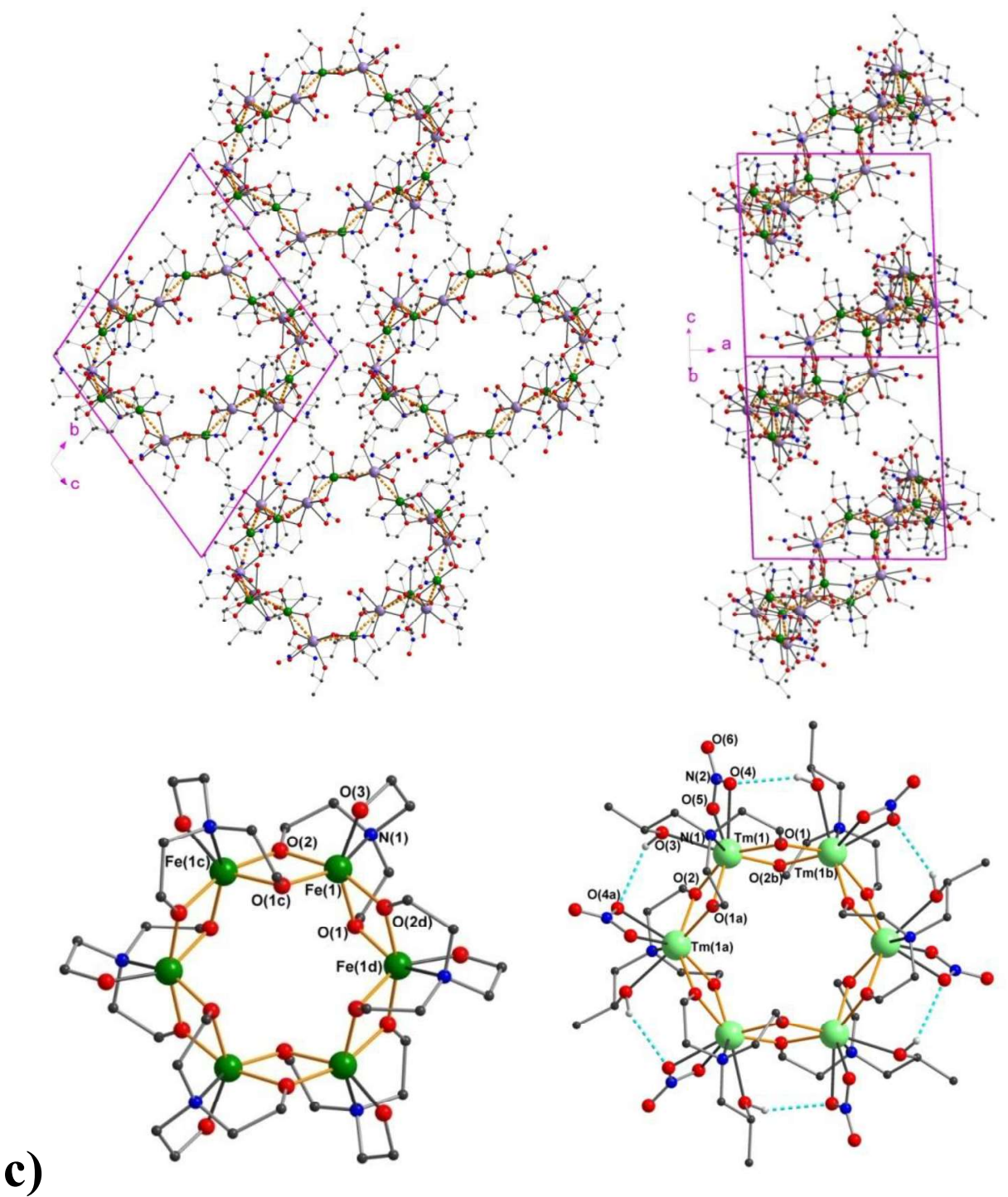
Submitted to

Figure 1. (a) Structure of the $\left[\mathrm{Fe}_{10} \mathrm{Ln}_{10}(\mathrm{Me} \text {-tea })_{10}(\mathrm{Me}-\mathrm{teaH})_{10}\left(\mathrm{NO}_{3}\right)_{10}\right]$ molecular magnet, projected along the normal to the torus mean plane; (b) Stacking of the $\mathrm{Fe}_{10} \mathrm{Ln}_{10}$ toruses in the crystal structure, viewed down the $a$-axis (left) and along the $\{011\}$ direction (right), showing the relatively weak overlap between the toruses; (c) Structure of $\left[\mathrm{Fe}_{6}(\text { tea })_{6}\right]$; (d) Structure of $\left[\mathrm{Tm}_{6}(\mathrm{Me}-\mathrm{teaH})_{6}\left(\mathrm{NO}_{3}\right)_{6}\right]$.

(Fe dark green, Dy violet, Tm light green, $\mathrm{O}$ red, $\mathrm{N}$ blue, $\mathrm{C}$ dark grey, H-bonds shown as blue dashed lines, "organic" $H$ atoms omitted for clarity. Symmetry equivalents a: $y+1 / 3$, $-\mathrm{x}+\mathrm{y}+2 / 3,-\mathrm{z}+2 / 3 ; \mathrm{b}: \mathrm{x}-\mathrm{y}+1 / 3, \mathrm{x}-1 / 3,-\mathrm{z}+2 / 3 ; \mathrm{c}: \mathrm{y},-\mathrm{x}+\mathrm{y},-\mathrm{z}+1 ; \mathrm{d}: \mathrm{x}-\mathrm{y}, \mathrm{x},-\mathrm{z}+1$

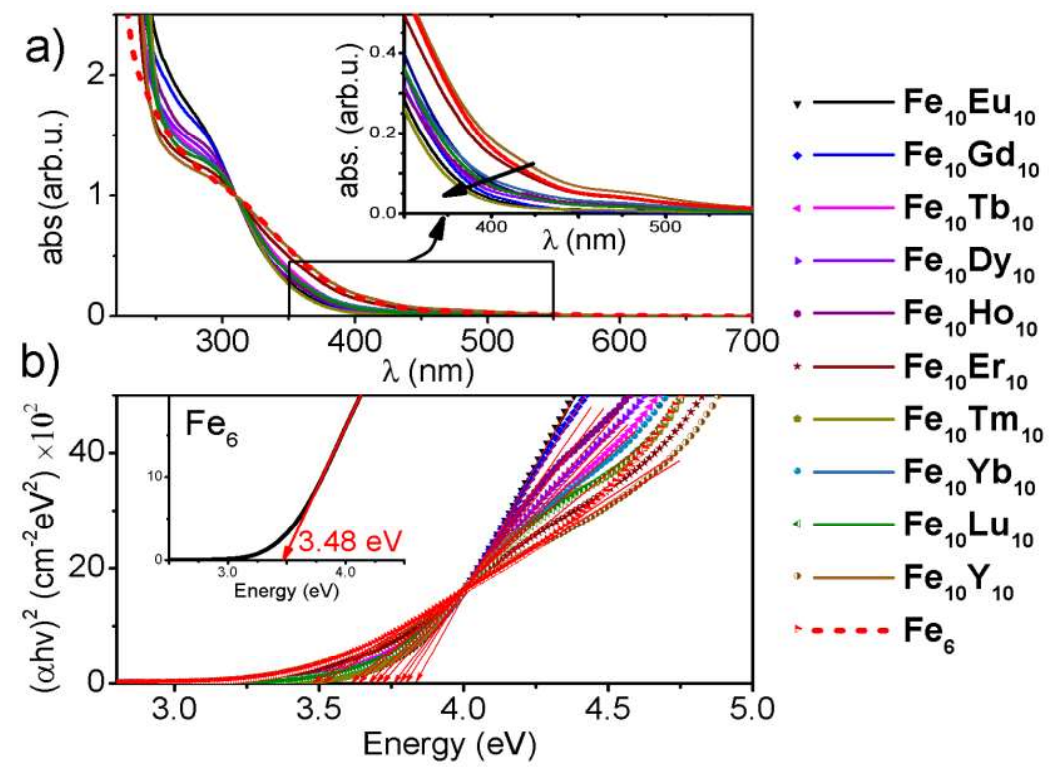

Figure 2. a) UV/Vis absorption spectra of $\mathrm{Fe}_{10} \mathrm{Ln}_{10}$ and $\mathrm{Fe}_{6}$ clusters, with absorbance renormalized by the value at $310 \mathrm{~nm}$. Inset shows the same data enlarged in order to exhibit the blue-shifted absorption edge of $\mathrm{Fe}_{10} \mathrm{Ln}_{10}$ clusters. The spectrum of $\mathrm{Fe}_{6}$ cluster is highlighted by red color. b) Optical energy gaps of $\mathrm{Fe}_{10} \mathrm{Ln}_{10}$ and $\mathrm{Fe}_{6}$ clusters estimated using Tauc equation. Inset shows the extrapolated optical energy gap of $3.48 \mathrm{eV}$ for $\mathrm{Fe}_{6}$ cluster. 
Submitted to

a)

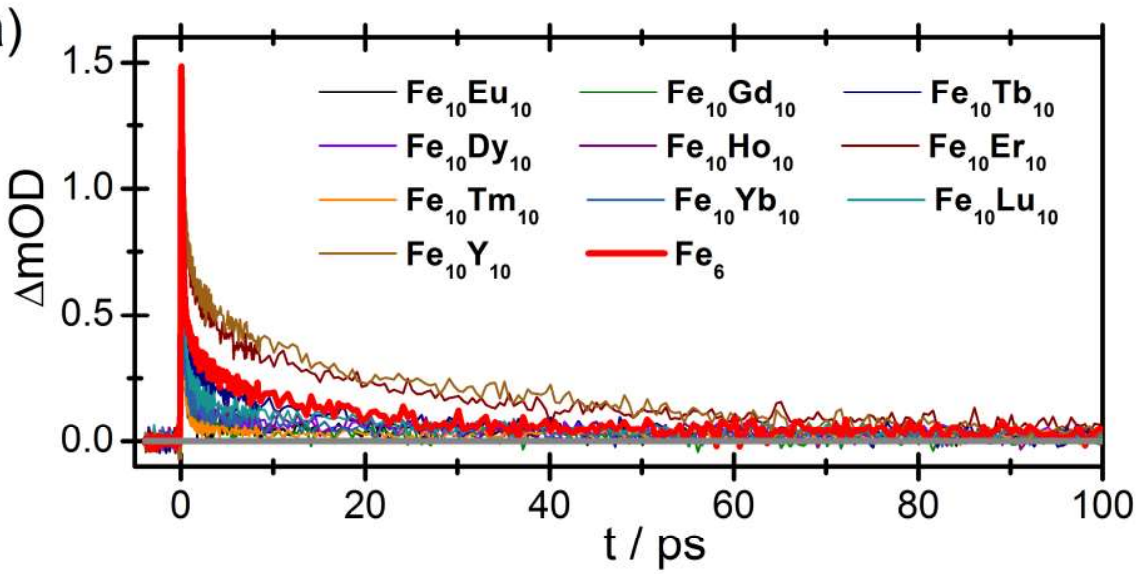

b)

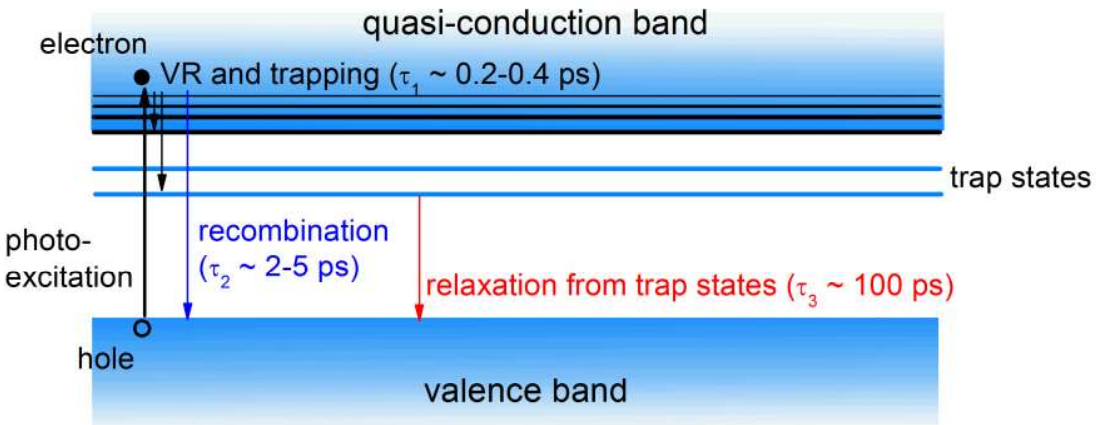

Figure 3. a) Time evolution of the transient absorption signal at $550 \mathrm{~nm}$ after excitation at $310 \mathrm{~nm}$, for all $\mathrm{Fe}_{10} \mathrm{Ln}_{10}$ and the $\mathrm{Fe}_{6}$ cluster in buffer solution, with the behavior of the $\mathrm{Fe}_{6}$ cluster highlighted in red. b) Simplified scheme for ultrafast relaxation dynamics in an iron oxo domain. (VR: vibrational relaxation) 


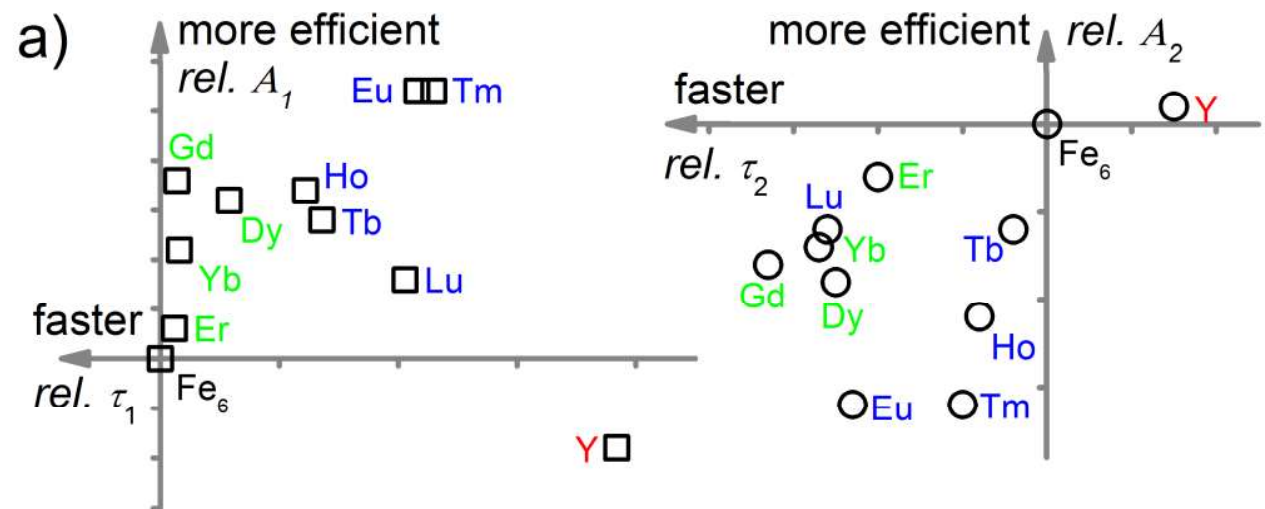

b)

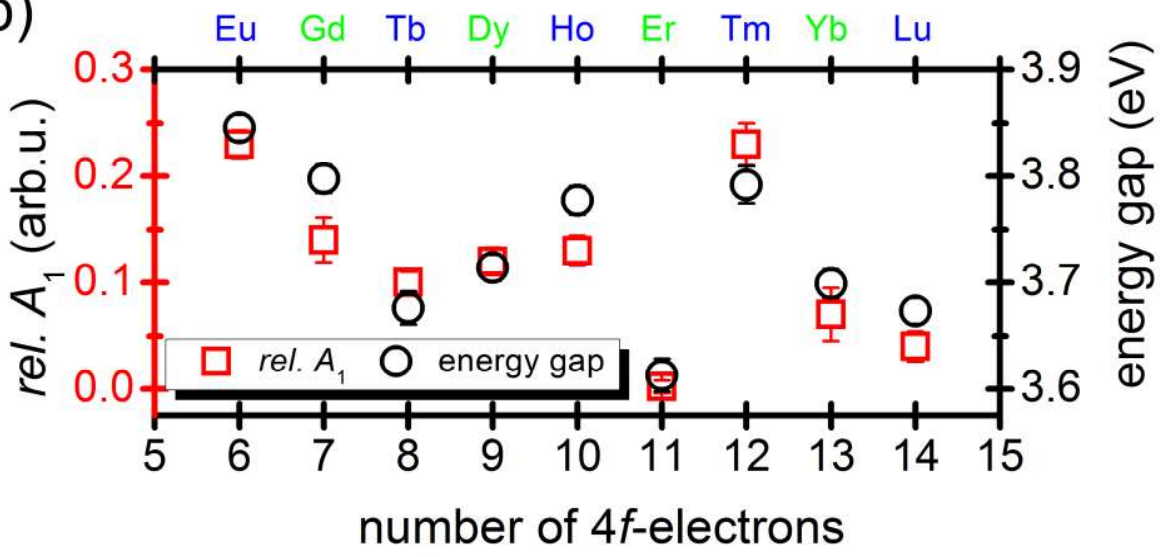

Figure 4. Plots of a) the relative amplitudes of the extracted relaxation processes vs their corresponding decay time, and b) the first relative amplitudes (squares) and the optical energy gaps (circles) of $\mathrm{Fe}_{10} \mathrm{Ln}_{10}$ versus the number of $4 \mathrm{f}$-electrons of the trivalent lanthanide ions. The reference cluster $\mathrm{Fe}_{6}$ set as zero reference point for sake of comparison. $\mathrm{Fe}_{10} \mathrm{Ln}_{10}$ with Kramers ions are shown as green and with non-Kramers ions as blue, $\mathrm{Fe}_{10} \mathrm{Y}_{10}$ in red.

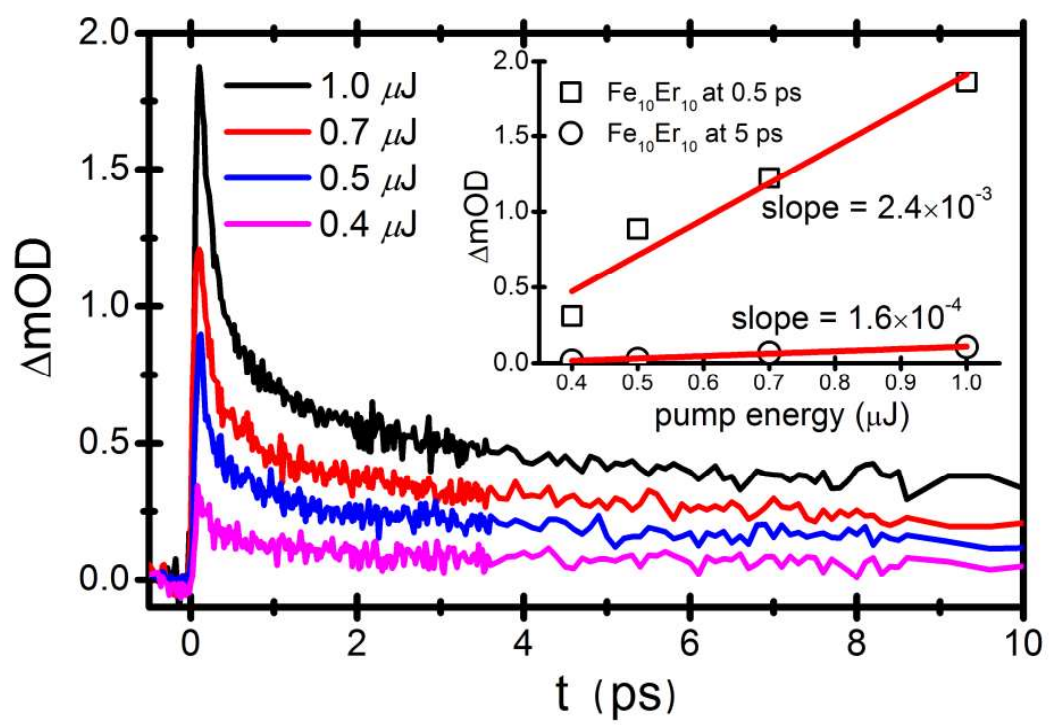

Figure 5. Transient absorption responses for $\mathrm{Fe}_{10} \mathrm{Er}_{10}$ at different excitation intensities. Inset shows the corresponding amplitudes of the transient absorption scale linearly to the excitation intensities. 
a)

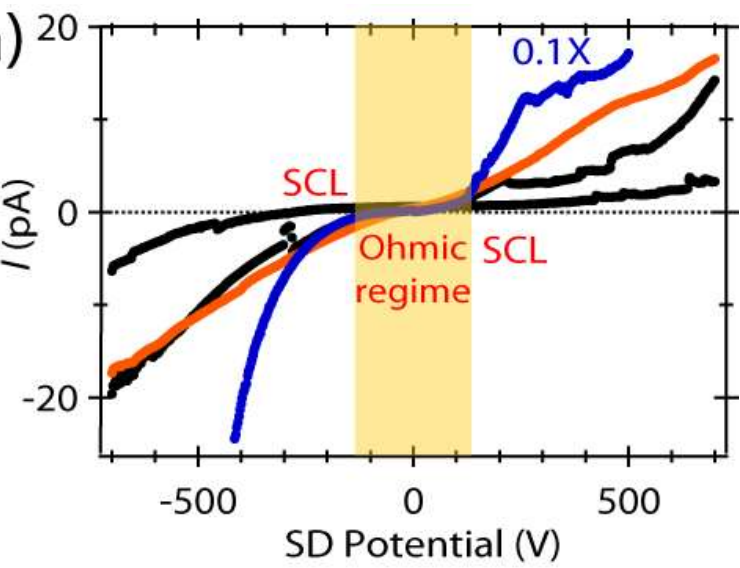

b)

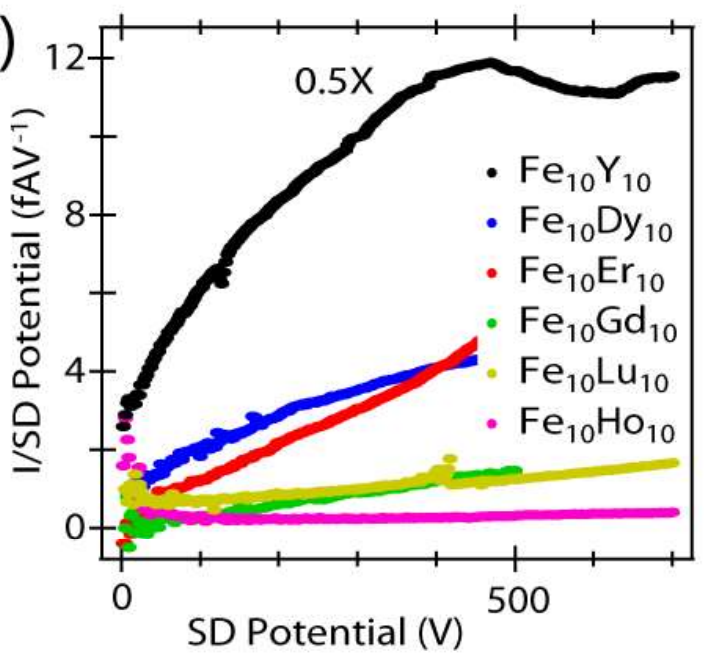

Figure 6. a) Current-voltage characteristics of several $\mathrm{Fe}_{10} \mathrm{Y}_{10}$ crystals, with black, red and blue curves from different crystals, and crystal A (black curve) contacted at two different positions; the linear ohmic region at low voltages is highlighted. b) Plot of $I / V$ vs $V$ for $\mathrm{Fe}_{10} \mathrm{Ln}_{10}$ crystals, highlighting the $\propto \quad 2$ dependence as straight lines. $\mathrm{Fe}_{10} \mathrm{Tm}_{10}$ and $\mathrm{Fe}_{10} \mathrm{~Tb}_{10}$ did not exhibit any measurable conductance (not shown). 
Submitted to

\section{ADVANCED FUNCTIONAL}

a) Intramolecular

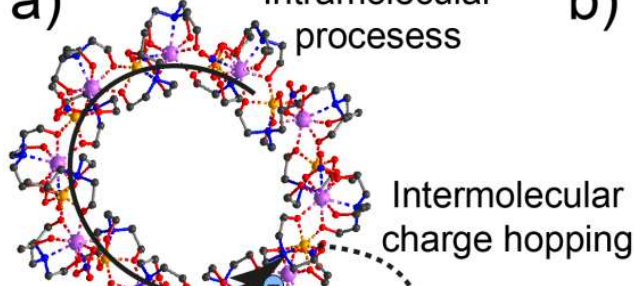

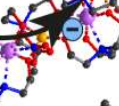
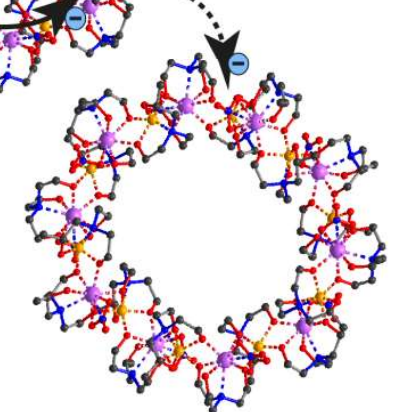

b)

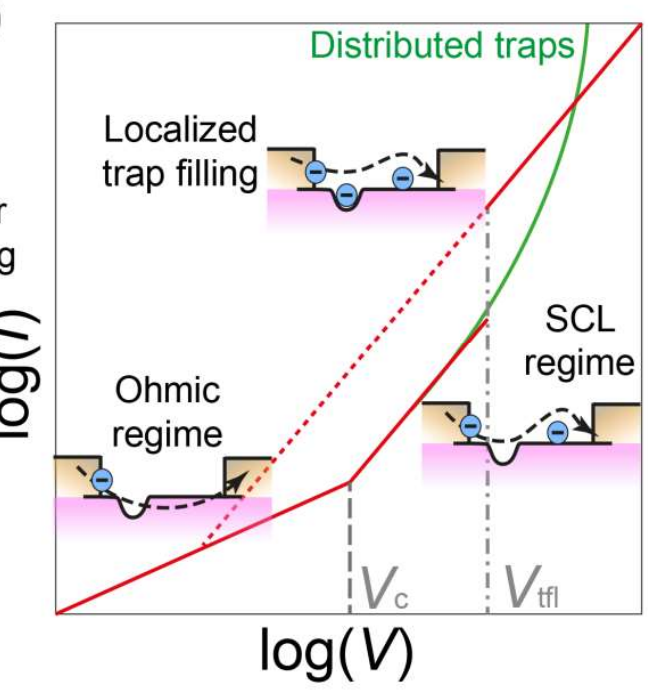

Figure 7. a) Scheme of the hopping transport mechanism, showing the intra- and intermolecular electron mobility. b) Expected behavior for space-charge limited transport, with the schematic representation of the relevant effects observed in different voltage regions. The effects of exponentially-distributed (green) and localized-energy (red) traps are also shown. 
Submitted to
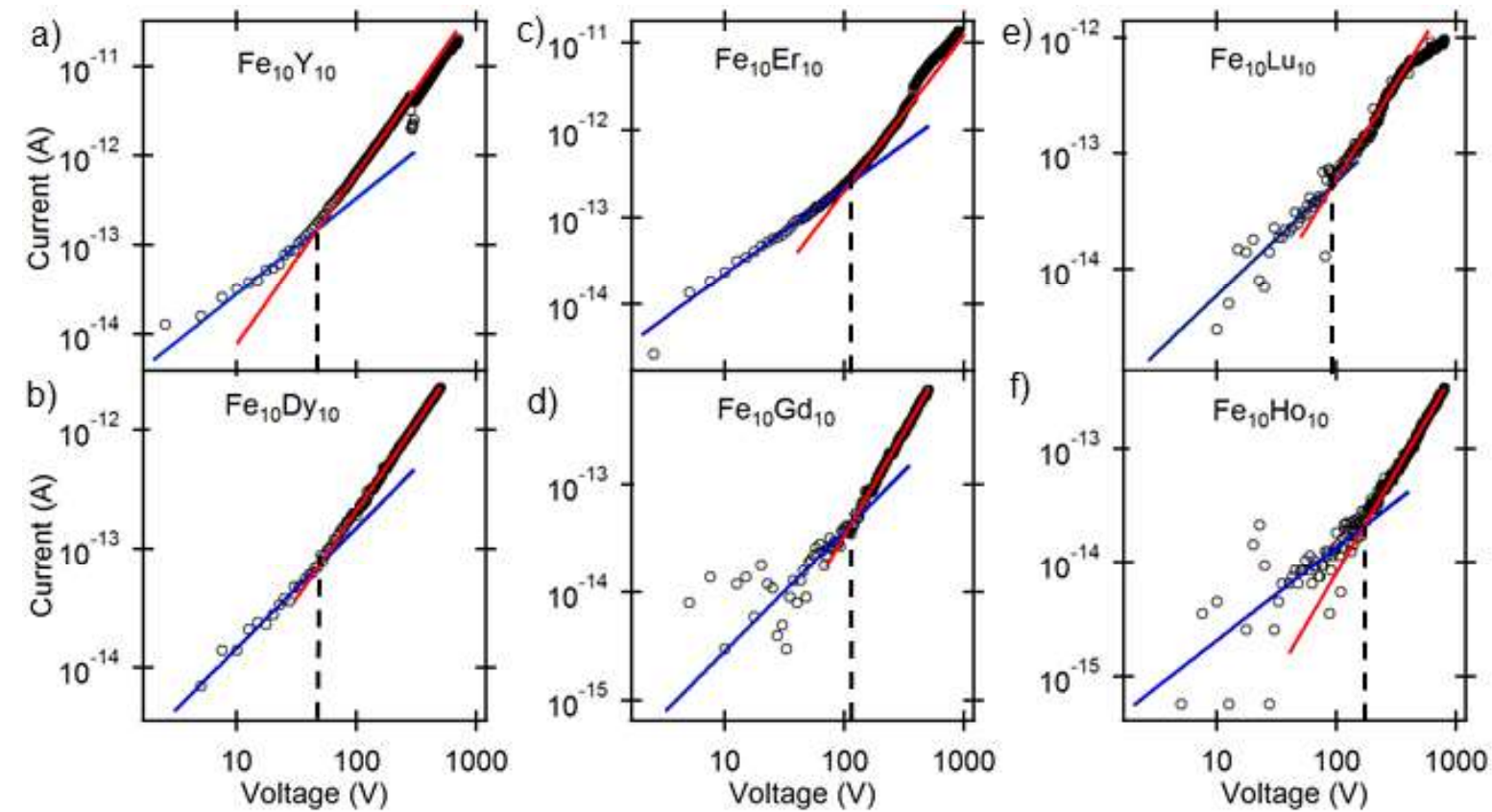

Figure 8. Log-log plot of the $I-V$-characteristics of $\mathrm{Fe}_{10} \mathrm{Ln}_{10}$. The ohmic (blue) and SCL (red) regimes are fitted with $=$. $\eta_{i}$ where i stands for the Ohmic $(\Omega)$ or charge-limited (SCL) regions (see Table 2). The crossover voltage $V_{c}$, obtained from the intersection of the two regimes, is highlighted with vertical dashed lines. See Table 2 for the extracted $n_{\text {th }}$ values. 
Submitted to
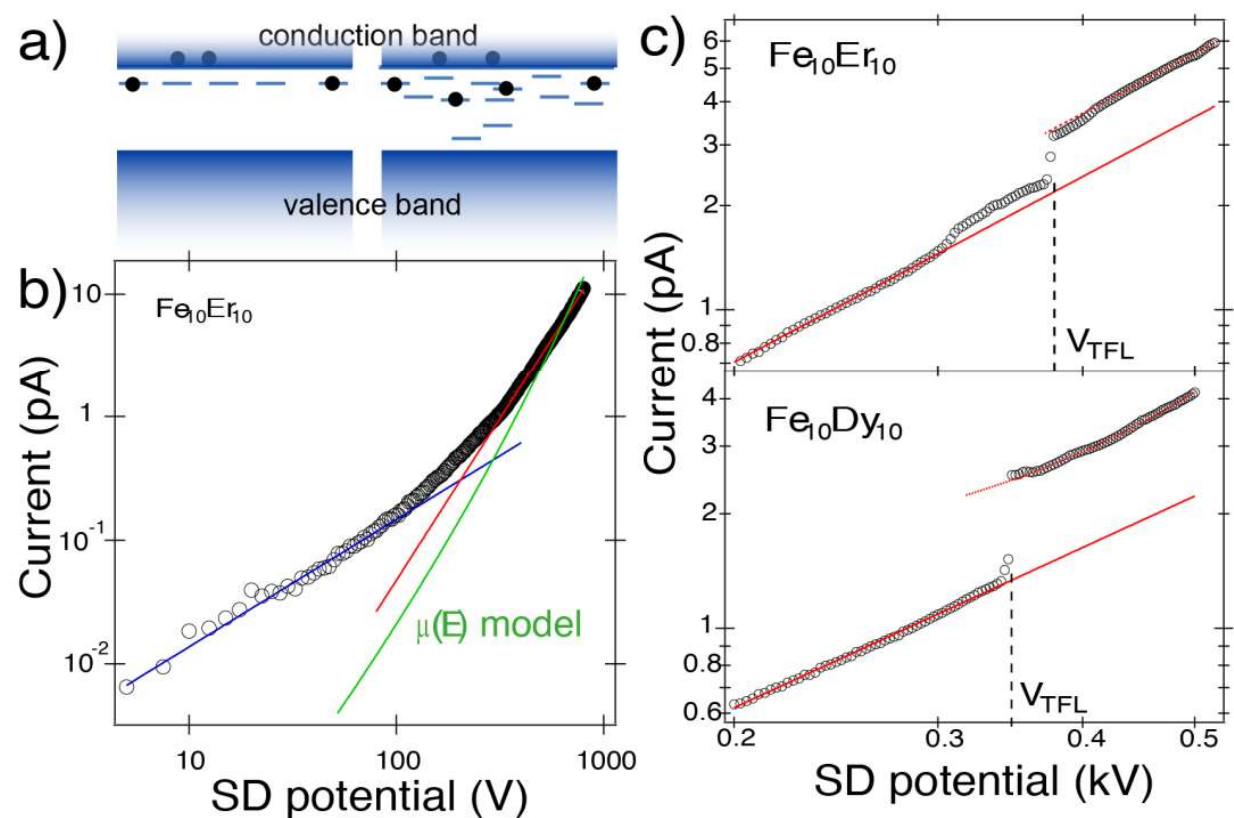

Figure 9. Examples of trapping effects, as observable in the $I$ - $V$-characteristics. a) Schematic depiction of localized traps (left) and energetically-distributed traps (right) placed between the valence and conduction bands of the system. b) exponentially-distributed traps in $\mathrm{Fe}_{10} \mathrm{Er}_{10}$ inducing a voltage dependence with an exponent larger than 2. Comparison between a trapfree SCL (red line) and a field-dependent mobility model with the behavior predicted by equation 6 (green line) is provided. c) Localized electron traps in $\mathrm{Fe}_{10} \mathrm{Er}_{10}$ (top) and $\mathrm{Fe}_{10} \mathrm{Dy}_{10}$ (bottom). The trapped-charge limited regime (solid red) and the trap-free SCL regime (dotted red) are shown, and the trap-filled limit $V_{\text {TFL }}$, is highlighted with dashed vertical lines. The lines are fits according to equation (4). 
Submitted to
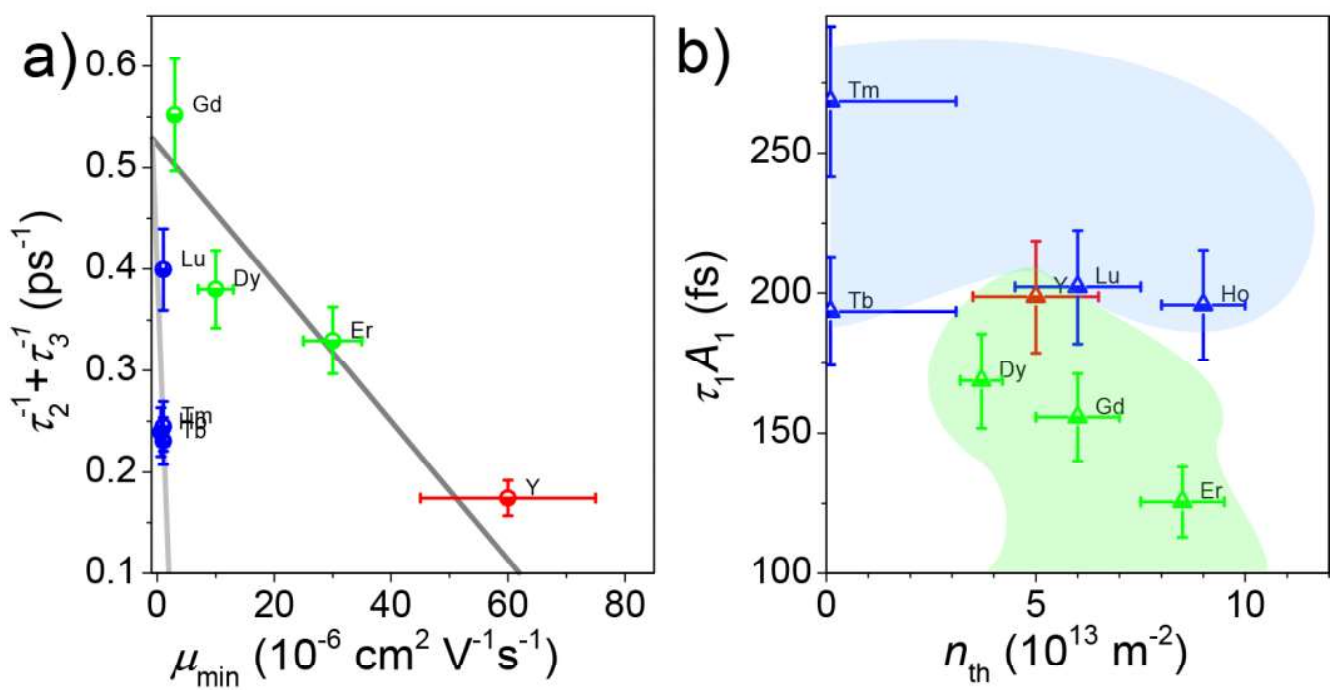

Figure 10. Observed relations between intra and intermolecular processes. a) Relation between the escape rate from the valence and shallow states and the transport mobility. Kramers ions (Gd, Dy, Er) are in green, non-Kramers ions (Tb, Ho, Tm, Lu) in blue, and the diamagnetic rare-earth $\mathrm{Y}$ in red; lines are guides to the eye. b) Dynamic/transport regions defined by Kramers and non-Kramers ions, showing also the absence of direct correlation between the trapping timescale $\tau_{1}$ and the carrier density at the crystal surface. 
Submitted to

Table 1. Optical energy gaps $\left(E_{\mathrm{opt}}\right)$, time constants $\left(\tau_{\mathrm{i}}\right)$ and corresponding relative amplitudes (rel. $A_{\mathrm{i}}$ ) of $\mathrm{Fe}_{10} \mathrm{Ln}_{10}$ and $\mathrm{Fe}_{6}$ clusters.

\begin{tabular}{cccccccc}
\hline Compound & $E_{\mathrm{opt}}[\mathrm{eV}]$ & $\mathrm{T}_{1}[\mathrm{fs}]$ & $\mathrm{T}_{2}[\mathrm{ps}]$ & $\mathrm{T}_{3}[\mathrm{ps}]$ & rel. $A_{1}$ & rel. $A_{2}$ & rel. $A_{3}$ \\
\hline $\mathrm{Fe}_{10} \mathrm{Eu}_{10}$ & $3.85 \pm 0.07$ & $298 \pm 31$ & $2.9 \pm 0.4$ & $121 \pm 19$ & 0.88 & 0.07 & 0.05 \\
$\mathrm{Fe}_{10} \mathrm{Gd}_{10}$ & $3.79 \pm 0.08$ & $197 \pm 18$ & $1.9 \pm 0.2$ & $39 \pm 6$ & 0.79 & 0.15 & 0.06 \\
$\mathrm{Fe}_{10} \mathrm{~Tb}_{10}$ & $3.7 \pm 0.1$ & $258 \pm 22$ & $4.8 \pm 0.6$ & $45 \pm 5$ & 0.75 & 0.17 & 0.08 \\
$\mathrm{Fe}_{10} \mathrm{Dy}_{10}$ & $3.71 \pm 0.04$ & $219 \pm 19$ & $2.7 \pm 0.3$ & $102 \pm 13$ & 0.77 & 0.14 & 0.09 \\
$\mathrm{Fe}_{10} \mathrm{Ho}_{10}$ & $3.77 \pm 0.05$ & $251 \pm 26$ & $4.4 \pm 0.6$ & $84 \pm 12$ & 0.78 & 0.12 & 0.10 \\
$\mathrm{Fe}_{10} \mathrm{Er}_{10}$ & $3.6 \pm 0.1$ & $196 \pm 18$ & $3.2 \pm 0.6$ & $58 \pm 10$ & 0.64 & 0.20 & 0.16 \\
$\mathrm{Fe}_{10} \mathrm{Tm}_{10}$ & $3.8 \pm 0.15$ & $305 \pm 30$ & $4.2 \pm 0.7$ & $150 \pm 22$ & 0.88 & 0.07 & 0.05 \\
$\mathrm{Fe}_{10} \mathrm{Yb}_{10}$ & $3.69 \pm 0.07$ & $198 \pm 21$ & $2.5 \pm 0.2$ & $74 \pm 12$ & 0.72 & 0.16 & 0.12 \\
$\mathrm{Fe}_{10} \mathrm{Lu}_{10}$ & $3.67 \pm 0.06$ & $293 \pm 24$ & $2.6 \pm 0.3$ & $67 \pm 6$ & 0.69 & 0.17 & 0.14 \\
$\mathrm{Fe}_{10} \mathrm{Y}_{10}$ & $3.51 \pm 0.09$ & $382 \pm 27$ & $6.7 \pm 1.2$ & $40 \pm 14$ & 0.52 & 0.24 & 0.24 \\
$\mathrm{Fe}_{6}$ & $3.48 \pm 0.03$ & $190 \pm 15$ & $5.2 \pm 1.4$ & $38 \pm 11$ & 0.61 & 0.23 & 0.16 \\
$\mathrm{Fe}_{7}$ & $\mathrm{~N} . \mathrm{A}$. & $367 \pm 16$ & $4.8 \pm 0.8$ & $57 \pm 8$ & 0.61 & 0.22 & 0.17 \\
\hline
\end{tabular}

Table 2. Minimum mobility $\mu_{\mathrm{min}}$, surface thermal carrier density $n_{\mathrm{th}}$, sheet resistance $R_{S}$, crossover voltage $V_{\mathrm{c}}$, Ohmic conductance $G_{\Omega}$, fitting exponent $\eta_{\Omega}$, and charge-limited parameters $G_{\mathrm{SCL}}$ and $\eta_{\mathrm{SCL}}$ and low-field mobility $\mu_{0}$, for the different rare-earth compounds.

\begin{tabular}{|c|c|c|c|c|c|c|c|c|c|}
\hline Compound & $\underset{\left[\mathrm{cm}^{2}(\mathrm{Vs})^{-1}\right]}{\mu_{\min }}$ & $\begin{array}{r}\operatorname{th}_{1 / 2}(2 \mathrm{D}) \\
{\left[10^{13} \mathrm{~m}^{-2}\right]}\end{array}$ & {$[\mathrm{T} \Omega]$} & $\begin{array}{l}V_{c} \\
{[\mathrm{~V}]}\end{array}$ & $\begin{array}{l}G_{\Omega} \\
{[S]}\end{array}$ & $\eta_{\Omega}$ & $\begin{array}{c}G_{S C L} \\
{\left[S \cdot V^{(n-1)}\right]}\end{array}$ & $\eta_{\mathrm{SCL}}$ & $\begin{array}{c}\mu_{0} \\
{\left[\mathrm{~cm}^{2}(\mathrm{Vs})^{-1}\right]}\end{array}$ \\
\hline $\mathrm{Fe}_{10} \mathrm{Y}_{10}$ & $6 \pm 2 \cdot 10^{-5}$ & $5 \pm 3$ & $20 \pm 10$ & 47 & $2.0 \pm 0.4 \cdot 10^{-15}$ & $1.1 \pm 0.1$ & $9.0 \pm 0.5 \cdot 10^{-17}$ & $1.9 \pm 0.1$ & $6 \cdot 10^{-5}$ \\
\hline $\mathrm{Fe}_{10} \mathrm{Er}_{10}$ & $3 \pm 1 \cdot 10^{-5}$ & $8.5 \pm 2$ & $57 \pm 9$ & 112 & $2.0 \pm 0.1 \cdot 10^{-15}$ & $1.0 \pm 0.1$ & $5.4 \pm 0.2 \cdot 10^{-17}$ & $1.8 \pm 0.1$ & $1.5 \pm 0.5 \cdot 10^{-5}$ \\
\hline $\mathrm{Fe}_{10} \mathrm{Dy}_{10}$ & $7 \pm 310^{-6}$ & $3.7 \pm 0.9$ & $72 \pm 5$ & 49 & $1.4 \pm 0.2 \cdot 10^{-15}$ & $1.0 \pm 0.1$ & $2.4 \pm 0.04 \cdot 10^{-16}$ & $1.5 \pm 0.1$ & $2.4 \pm 0.9 \cdot 10^{-5}$ \\
\hline $\mathrm{Fe}_{10} \mathrm{Gd}_{10}$ & $3 \pm 1 \cdot 10^{-6}$ & $6 \pm 2$ & $280 \pm 40$ & 112 & $2.2 \pm 0.8 \cdot 10^{-16}$ & $1.1 \pm 0.1$ & $4.9 \pm 0.3 \cdot 10^{-18}$ & $1.9 \pm 0.1$ & $4.5 \pm 2 \cdot 10^{-6}$ \\
\hline $\mathrm{Fe}_{10} \mathrm{Lu}_{10}$ & $1 \pm 0.5 \cdot 10^{-6}$ & $6 \pm 3$ & $115 \pm 4$ & 110 & $6 \pm 2 \cdot 10^{-16}$ & $1.0 \pm 0.1$ & $2.7 \pm 0.4 \cdot 10^{-17}$ & $1.7 \pm 0.2$ & $10 \pm 4 \cdot 10^{-6}$ \\
\hline $\mathrm{Fe}_{10} \mathrm{Ho}_{10}$ & $6.3 \pm 0.1 \cdot 10^{-7}$ & $9 \pm 2$ & $620 \pm 80$ & 170 & $3 \pm 2 \cdot 10^{-16}$ & $0.8 \pm 0.1$ & $2.2 \pm 0.1 \cdot 10^{-18}$ & $1.8 \pm 0.1$ & $1.2 \pm 0.2 \cdot 10^{-6}$ \\
\hline
\end{tabular}


Table of Contents entry:

Submitted to

Inter- and intra-molecular electron-transport processes are studied for a family of lanthanidebased molecular toruses using ultrafast-optical and electron-transport techniques. A relationship between trap efficiency and gaps is found, and charge-hopping and the dominant role of surface-limited transport is established. Comparison between intra- and intermolecular processes highlights the importance of excited electronic states, showing the influence of Kramers' parity on mobility.

Keywords: lanthanides, electron transport, femtosecond spectroscopy, cyclic coordination clusters, molecular magnetism.

Amer Baniodeh $^{1,2}$, Yu Liang ${ }^{3}$, Christopher E. Anson ${ }^{2}$, Nicola Magnani ${ }^{1}$, Annie K. Powell $^{1,2 *}$, Andreas-Neil Unterreiner ${ }^{3, *}$, Simon Seyfferle ${ }^{4}$, Michael Slota ${ }^{4}$, Martin Dressel $^{4}$, Lapo Bogani ${ }^{4}$, Karin Goß ${ }^{4, *}$

Unraveling the Influence of Lanthanide Ions on Intra- and Inter-Molecular Electronic Processes in $\mathrm{Fe}_{10} \mathrm{Ln}_{10}$ nano-Toruses

ToC figure:

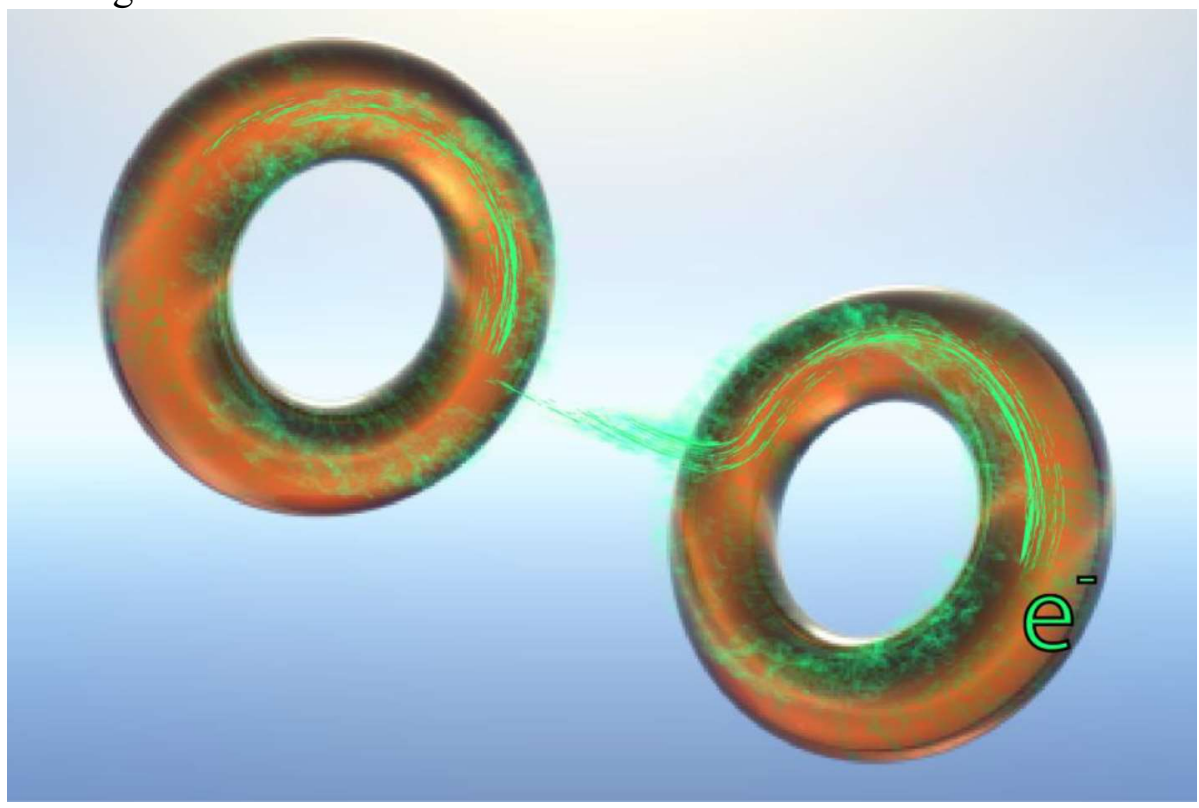

